\title{
Digging for top squarks from Higgs data: from signal strengths to differential distributions
}

\author{
Andrea Banfi, ${ }^{a}$ Andrew Bond, ${ }^{a}$ Adam Martin ${ }^{b}$ and Verónica Sanz ${ }^{a}$ \\ ${ }^{a}$ Department of Physics and Astronomy, University of Sussex, \\ Brighton BN1 9QH, U.K. \\ ${ }^{b}$ Department of Physics, University of Notre Dame, \\ Notre Dame, IN 46556, U.S.A. \\ E-mail: a.banfi@sussex.ac.uk, a.bond@sussex.ac.uk, amarti41@nd.edu, \\ v.sanz@sussex.ac.uk
}

ABSTRACT: One way to hunt for top squarks is to look for deviations from the Standard Model in loop level processes involving Higgses. This method is indirect, but complementary to direct searches as it does not rely on specific top squark decays. Studying inclusive Higgs production $p p \rightarrow h$ alone is insufficient, since there are parameter regions where the effects of the two top squarks approximately cancel. This degeneracy can be broken by looking at the rate for highly boosted Higgses recoiling against a jet, $p p \rightarrow h+$ jet. In this paper we perform a detailed study of the complementarity of the inclusive and highly boosted processes at the LHC, both in existing Run 1 and Run 2 data, and looking forward to high luminosity. To break the degeneracy, our calculation must maintain the full mass dependence in the loop functions and therefore cannot be recast in an effective field theory framework. We quantify the dependence of both topologies in the top squark parameter space, and outline which levels of experimental and theoretical understanding would be needed for boosted Higgses to be competitive with inclusive Higgs production.

KeYwORDS: Jets, Supersymmetry Phenomenology

ARXiv EPrint: 1806.05598 


\section{Contents}

1 Introduction 1

2 Top squarks and the Higgs $\quad 2$

2.1 Top squark contributions to $g g \rightarrow h \quad 4$

2.2 Top squark contributions to $p p \rightarrow h+$ jet 5

$\begin{array}{lll}3 & \text { Numerical results } & 9\end{array}$

4 Comparing inclusive Higgs and high- $p_{T}$ Higgs sensitivities 13

5 Discussion and outlook $\quad 16$

$\begin{array}{ll}\text { A Higgs plus one jet for large top squark masses } & 18\end{array}$

$\begin{array}{lr}\text { B Higgs plus one jet in the soft and collinear limit } & 18\end{array}$

$\begin{array}{lll}\text { B.1 Soft limit } & 19\end{array}$

B.2 Collinear limits 20

\section{Introduction}

The second phase of the LHC is well underway and will usher in the era of precision Higgs physics, hunting for any sign of a deviation from the Standard Model (SM) expectations. Being a hadron collider, collisions at the LHC are inherently chaotic and complicated by low-energy QCD effects. This ultimately limits the accuracy at which particle properties can be measured. Future lepton colliders, such as CEPC, FCC-ee, and ILC, CLIC and TLEP [1-9] offer a cleaner environment and improved precision, but none of the currently discussed possibilities possess the energy of the LHC.

In this paper we study the interplay between the two approaches, precision and energy. As our testing ground, we will use the top squark sector of the minimal supersymmetric standard model (MSSM). Top squarks modify Higgs properties at loop level [10-14], and the size and nature of the effects therefore depend on the energies involved. At low energies (on-shell Higgs), top squark loops will modify both Higgs production and decay, which we encapsulate using signal strengths. For our high-energy observable we will look at Higgs plus jet production.

While top squarks can be searched for directly [15, 16], direct searches rely on assumptions about the decay products, branching ratios, and spectrum. Direct searches for stops are particularly sensitive to the assumption of R-parity conservation in Supersymmetry, and whether the spectrum is well spaced. If R-parity were not a good symmetry of low-energy one would expect the stop to decay into complex final states, probably not 
involving a large quantity of missing energy as usually assumed. But even if R-parity was a good symmetry and stops decays end in a neutral and stable state, the spectrum and couplings could be such that stop decays are an admixture of several decay channels, again weakening the direct stop sensitivity.

On the other hand, indirect probes of the top squark sector, such as Higgs coupling measurements, are based on different assumptions and hence complementary to direct searches. For example, effects of top squarks on Higgs production are independent of the rest of the spectrum and fixed to a large extent by supersymmetry, although related to the sbottom mass spectrum and direct searches [17]. Nevertheless, the interpretation of Higgs couplings in terms of solely stop contributions assumes no other sector, e.g. sbottoms or some new coloured states, leads to a competing contribution.

Top squark effects in Higgs plus jet production have been explored previously in ref. [18], where the authors focused on the region where the stop effects cancel in inclusive production. In ref. [19], the differential rates of Higgs plus jets where discussed in the context of the MSSM, but no explicit expressions or analytical discussion the differential rates was given. Nowadays, a tool called SusHi [20-22] allows the user to compute differential rates and interface with Monte Carlo generators such as aMC@NLO [23]. Compared to that work, the present study is more comprehensive, covering a wider array of top squark scenarios and comparing the top squark sensitivity of inclusive Higgs cross section measurements with that of highly boosted Higgses. We also provide a more detailed discussion of the calculation of Higgs plus jet with scalar contributions, with cross checks in the soft and collinear limits. Our differential rates are computed using a fast Gaussian integrator, allowing the user to obtain the differential rates in a fraction of seconds, hence it is useful when dealing with spectrum parameter scans. There are also several studies of generic new physics loops affecting Higgs production within the framework of effective field theory [24-29] both at leading and next-to-leading (NLO) accuracy, where by definition the mass of the new particles is assumed to be large compared to the momentum of the process. Our study includes the full superpartner mass dependence. As we will show, this additional information allows us to differentiate between supersymmetric spectra that are degenerate when $\sqrt{\hat{s}} \ll m_{N P}$. The utility of Higgs plus jet has been explored in the past in a similar fashion to find fermionic top-partners [18, 30-32].

The setup of this paper is as follows: in section 2 we define the top squark parameters and explore their contribution to inclusive Higgs production. Next, in section 2.2, we introduce Higgs plus jet production and present our analytic results. The full form of the scalar loop contribution to Higgs plus jet is postponed to appendix A along with several crosschecks in special kinematic regions. In section 3 and section 4, we introduce the simulation tools and numerically explore the complementarity between inclusive Higgs production and Higgs plus jet for exposing top squark signals. Finally, in section 5, we conclude.

\section{Top squarks and the Higgs}

While the minimal supersymmetric Standard Model (MSSM) is a vast framework with rich phenomenology, for the purposes of this work the only aspects of the MSSM that we care about is the top squark couplings to the Higgs and their masses. As such, we 
are not interested in features that require knowledge of the complete spectrum, such as how the measured Higgs mass is achieved or how/whether there is a viable dark matter candidate. The top squark sector of the MSSM consists of two complex scalar fields, $\tilde{t}_{L}, \tilde{t}_{R}$, both of which receive the bulk of their mass from supersymmetry breaking. In addition to $\left|\tilde{t}_{L}\right|^{2},\left|\tilde{t}_{R}\right|^{2}$ type masses, the two scalars can mix through interaction with one of the MSSM Higgses. Rather than working with the entries of the top squark mass matrix, we will parameterize the stop sector by:

$$
\left(m_{\tilde{t}_{1}}, \Delta m, \theta\right),
$$

where the lightest top squark mass is denoted by $m_{\tilde{t}_{1}}$, the mixing angle by $\theta$ which lies in the interval $[-\pi / 2, \pi, 2]$, and $\Delta m$ is the separation with the next state, $\Delta m^{2}=m_{\tilde{t}_{2}}^{2}-m_{\tilde{t}_{1}}^{2}$.

With this parametrization, one can write the coupling of the lightest Higgs boson to the mass eigenstate top squarks as

$$
\begin{aligned}
& g_{h \tilde{t}_{1} \tilde{t}_{1}}=\frac{m_{t}^{2}}{v}\left(\alpha_{1} c_{\theta}^{2}+\alpha_{2} s_{\theta}^{2}+\frac{c_{\alpha}}{s_{\beta}}\left(2-\frac{\Delta m^{2}}{2 m_{t}^{2}} s_{2 \theta}^{2}\right)+\frac{c_{\alpha-\beta}}{s_{\beta}^{2}} \frac{\mu}{m_{t}} s_{2 \theta}\right), \\
& g_{h \tilde{t}_{2} \tilde{t}_{2}}=\frac{m_{t}^{2}}{v}\left(\alpha_{1} s_{\theta}^{2}+\alpha_{2} c_{\theta}^{2}+\frac{c_{\alpha}}{s_{\beta}}\left(2+\frac{\Delta m^{2}}{2 m_{t}^{2}} s_{2 \theta}^{2}\right)-\frac{c_{\alpha-\beta}}{s_{\beta}^{2}} \frac{\mu}{m_{t}} s_{2 \theta}\right),
\end{aligned}
$$

where

$$
\begin{aligned}
\alpha_{1} & =-\frac{m_{Z}^{2}}{m_{t}^{2}}\left(1-\frac{4}{3} s_{W}^{2}\right) s_{\alpha+\beta}, \\
\alpha_{2} & =-\frac{4}{3} \frac{m_{Z}^{2}}{m_{t}^{2}} s_{W}^{2} s_{\alpha+\beta},
\end{aligned}
$$

and $v=2 m_{W} / g \simeq 246 \mathrm{GeV}$. Here we use the shorthand notation $s_{\beta} \equiv \sin \beta, c_{\beta} \equiv$ $\cos \beta, s_{W} \equiv \sin \theta_{W}$, etc., where $\tan \beta$ is the ratio of the two Higgs vevs and $\alpha$ is the mixing angle rotating the CP-even neutral components of the two Higgses to the mass eigenstates, $h$ and $H .{ }^{1}$ In the decoupling limit [33], valid as long as the mass of the pseudoscalar $A$ is large compared to the weak scale $\left(m_{A} \gg m_{Z}\right)$, the angles $\alpha$ and $\beta$ are related by $\alpha=\beta-\frac{\pi}{2}$ and the couplings simplify to

$$
\begin{aligned}
& g_{h \tilde{t}_{1} \tilde{t}_{1}}=\frac{m_{t}^{2}}{v}\left(\alpha_{1} c_{\theta}^{2}+\alpha_{2} s_{\theta}^{2}+2-\frac{\Delta m^{2}}{2 m_{t}^{2}} s_{2 \theta}^{2}\right), \\
& g_{h \tilde{t}_{2} \tilde{t}_{2}}=\frac{m_{t}^{2}}{v}\left(\alpha_{1} s_{\theta}^{2}+\alpha_{2} c_{\theta}^{2}+2+\frac{\Delta m^{2}}{2 m_{t}^{2}} s_{2 \theta}^{2}\right),
\end{aligned}
$$

where the coefficients $\alpha_{1,2}$ reduce to

$$
\begin{aligned}
\alpha_{1} & =\frac{m_{Z}^{2}}{m_{t}^{2}} c_{2 \beta}\left(1-\frac{4}{3} s_{W}^{2}\right), \\
\alpha_{2} & =\frac{4}{3} \frac{m_{Z}^{2}}{m_{t}^{2}} c_{2 \beta} s_{W}^{2} .
\end{aligned}
$$

We will assume $m_{A} \gg m_{Z}$ throughout this paper.

\footnotetext{
${ }^{1}$ In addition to $g_{h \tilde{t}_{1} \tilde{t}_{1}}, g_{h \tilde{t}_{2} \tilde{t}_{2}}$ there is a mixed coupling $g_{h \tilde{t}_{1} \tilde{t}_{2}}$. We will ignore this coupling throughout since it cannot lead to a 1-loop contribution to $p p \rightarrow h$ or $p p \rightarrow h+$ jet.
} 
Having defined how the top squarks couple to the lightest Higgs boson, we next study their impact on inclusive Higgs production and Higgs plus jet production. In both cases, the top squarks enter at loop level, induced by gluons and/or quarks. As the stop-gluon coupling is fixed by $\mathrm{SU}(3)$ invariance, the top squark contribution to Higgs (and Higgs + jet) production for a given $\hat{s}$ is a function of the stop masses and mixing alone.

\subsection{Top squark contributions to $g g \rightarrow h$}

Focusing first on Higgs production via gluon fusion, the ratio of cross section in the MSSM to the cross section in the SM is given by [34-37]

$$
\frac{\sigma_{\mathrm{MSSM}}(g g \rightarrow h)}{\sigma^{\mathrm{SM}}(g g \rightarrow h)} \simeq \frac{\Gamma(h \rightarrow g g)}{\Gamma^{\mathrm{SM}}(h \rightarrow g g)} \equiv \kappa_{g}^{2} \simeq\left(1+\frac{C_{g}\left(\alpha_{s}\right) F_{g}\left(m_{\tilde{t}_{1}}, m_{\tilde{t}_{2}}, \theta_{\tilde{t}}\right)}{F_{g}^{\mathrm{SM}}\left(m_{t}, m_{b} \cdots\right)}\right)^{2} .
$$

The function $F_{g}$ depend on the masses of particles in the loop and their couplings to the Higgs. For the SM, the only important contribution is the top quark, while in the MSSM loops of both types of top squarks will contribute. ${ }^{2}$ Both the MSSM and SM contributions receive higher order QCD corrections. As these corrections are not the same, there is some residual effect after taking the ratio which we encapsulate into the factor $C_{g}$. Expanding out the mass and coupling dependence of $F_{g}$ for the cases of interest:

$$
\begin{aligned}
F_{g}\left(m_{\tilde{t}_{1}}, m_{\tilde{t}_{2}}, \theta_{\tilde{t}}\right) & =\sum_{i=\tilde{t}_{1}, \tilde{t}_{2} \cdots} g_{h \tilde{t}_{i} \tilde{t}_{i}} \frac{v}{2 m_{i}^{2}} F_{0}\left(\tau_{i}\right), \\
F_{g}^{\mathrm{SM}}\left(m_{t}, m_{b} \cdots\right) & =\sum_{i=t, b \cdots} F_{1 / 2}\left(\tau_{i}\right)\left(1+\frac{11 \alpha_{s}}{4 \pi}\right) .
\end{aligned}
$$

For a given particle $i$ running around the gluon fusion loop, the functions $F_{0}$ and $F_{1 / 2}$ depend on the dimensionless variable $\tau_{i}=m_{h}^{2} /\left(4 m_{i}^{2}\right)$ and can be further decomposed as:

$$
\begin{aligned}
F_{0}(\tau) & =[\tau-f(\tau)] / \tau^{2}, \quad F_{1 / 2}(\tau)=-2[\tau+(\tau-1) f(\tau)] / \tau^{2}, \\
\text { with } \quad f(\tau) & =\left\{\begin{array}{lc}
\arcsin ^{2} \sqrt{\tau} & \tau \leq 1 \\
-\frac{1}{4}\left[\log \frac{1+\sqrt{1-\tau^{-1}}}{1-\sqrt{1-\tau^{-1}}}-i \pi\right]^{2} & \tau>1
\end{array} .\right.
\end{aligned}
$$

To gain some insight into eq. (2.11), it is useful to take some limits. If both the top squarks are heavy, $m_{\tilde{t}_{1,2}} \gg m_{h}$, the top squark contribution can be matched onto local operators in the context of an EFT analyses [38-41], and the functions $F_{g}, C_{g}$ simplify. Neglecting the effects of gluinos, squark mixing, and any running that would sum the large logs that would appear if the matching was done in two stages (e.g. one top squark eigenstate at a time), the perturbative matching correction is [42]:

$$
C_{g}\left(\alpha_{s}\right)=1+\frac{25 \alpha_{s}}{6 \pi}
$$

\footnotetext{
${ }^{2}$ In this comparison we are neglecting subdominant contributions from other squarks.
} 
Carrying out $m_{\tilde{t}_{1,2}} \gg m_{h}$ in $F_{g}\left(m_{\tilde{t}_{1}}, m_{\tilde{t}_{2}}, \theta_{\tilde{t}}\right)$, we find

$$
\begin{aligned}
F_{g}\left(m_{\tilde{t}_{1}}, m_{\tilde{t}_{2}}, \theta_{\tilde{t}}\right) & =-\frac{1}{3} \sum_{i=\tilde{t}_{1}, \tilde{t}_{2}} \frac{g_{h \tilde{t}_{t_{i}} \tilde{t}_{i}} v}{2 m_{i}^{2}} \\
& =-\frac{1}{3}\left[\frac{m_{t}^{2}}{m_{\tilde{t}_{1}}^{2}}+\frac{m_{t}^{2}}{m_{\tilde{t}_{2}}^{2}}-\frac{1}{4} \sin ^{2}(2 \theta) \frac{\Delta m^{4}}{m_{\tilde{t}_{1}}^{2} m_{\tilde{t}_{2}}^{2}}\right] .
\end{aligned}
$$

up to corrections of $\mathcal{O}\left(g^{2}\right)$. Clearly, eq. (2.15) is the sum of two types of terms, a positivedefinite contribution from both mass eigenstates, and another dependent on the mixing angle. As such, the size - and even overall sign — of $F_{g}\left(m_{\tilde{t}_{1}}, m_{\tilde{t}_{2}}, \theta_{\tilde{t}}\right)$ depends on the details of the mixing in the stop sector. The stop contribution depends on the mass and chirality of the eigenstates. If the overall contribution is dominated by a light eigenstate of pure handedness $(\theta \rightarrow 0)$ the sum will be negative, whereas if the mixing term proportional to $\Delta m^{2}$ is dominant then the sum will be positive. Stated another way, in the case of zero mixing we expect there to be an enhancement of $\sigma(g g \rightarrow h)$, but in the case where there is sizable mixing and the $\Delta m^{2}$ term dominates, the separation between the two eigenstates will govern the suppression. Dialling the mixing between the $\theta \rightarrow 0$ limit and the limit of large $\Delta m^{2}$, it is clear that there are slices of parameter space where the contributions from the two states partially cancel each other and $F_{g}\left(m_{\tilde{t}_{1}}, m_{\tilde{t}_{2}}, \theta_{\tilde{t}}\right) \sim 0$. For these parameter regions, $g g \rightarrow h$ will have reduced sensitivity of the top squark sector. We emphasize that, while the possibility of cancellation in $F_{g}$ when there is large mixing among the top squarks is easiest to see analytically in the $m_{\tilde{t}_{1}}, m_{\tilde{t}_{2}} \gg m_{t}$ limit, it is not restricted to that parameter region.

As we will show below, by adding an extra jet to the final state $p p \rightarrow h+$ jet and focusing on regions where the Higgs has high $p_{T}$, we can disrupt the cancellation among top squark loops. Whereas these cancellations may be accidental, and hence unmotivated from a model building point of view, adding differential information from $p p \rightarrow h+$ jet can only increase the amount of information we have on the stop sector. Moreover, future measurements of deviations in both total rates and differential rates may allow us to distinguish among different types of stop sectors, pointing out to specific UV realizations of supersymmetry.

\subsection{Top squark contributions to $p p \rightarrow h+$ jet}

Higgs plus jet production in the SM is a one-loop process induced by $g g, q g, \bar{q} g$ or $\bar{q} q$ partons. The top squark contribution has been calculated previously in refs. [18-22]; however this is the first instance where the analytic form of the amplitude is given. The analytic form is useful as it allows us to understand how the contributions behave in different kinematic limits. In addition to the analytic expression for the top squark contributions, we also show their behaviour in the soft and collinear limits in appendix B. While not strictly required for our numerical analysis, the soft and collinear limits serve as a valuable cross-check.

The $g g \rightarrow h g$ amplitude can be expressed in terms of eight primitive helicity amplitudes $\mathcal{M}_{h_{1} h_{2} h_{3}}$ corresponding to the possible choices for each gluon helicity $h_{i}= \pm$. We use the convention that the momenta of gluons $p_{1}$ and $p_{2}$ are incoming, and that of gluon $p_{3}$ is 
outgoing, so that the Mandelstam variables are defined as

$$
s=\left(p_{1}+p_{2}\right)^{2}, \quad t=\left(p_{2}-p_{3}\right)^{2}, \quad u=\left(p_{1}-p_{3}\right)^{2} .
$$

The helicity amplitudes are then related to the full, un-averaged amplitude squared via

$$
\left|M_{g g \rightarrow H g}\right|^{2}=\frac{N_{c}\left(N_{c}^{2}-1\right) \alpha_{s}^{3}}{64 \pi v^{2}} \sum_{h_{1}, h_{2}, h_{3}= \pm}\left|\sum_{i=t, b, \tilde{t}_{1}, \tilde{t}_{2}} \mathcal{M}_{h_{1} h_{2} h_{3}}^{i}\right|^{2} .
$$

The index $i$ here refers to the particle running in the loop needed to couple the gluons to the Higgs. After applying parity and crossing symmetry, only two of the helicity amplitudes are independent, which we take to be $\mathcal{M}_{+++}^{i}$ and $\mathcal{M}_{++-}^{i}$. The amplitudes for fermions in the loops (needed for $i=t, b$ ) can be found in appendix A of ref. [45].

The contributions to the helicity amplitudes due to loops containing a top squark with mass $m=m_{\tilde{t}_{i}}$ and coupling to the Higgs $g_{h \tilde{t}_{i} \tilde{t}_{i}}$, are:

$$
\begin{aligned}
\mathcal{M}_{+++}^{\tilde{t}_{i}}= & \left(g_{h \tilde{t}_{i} \tilde{t}_{i}} v \Delta\right) \times\left\{16\left(\frac{1}{t u}+\frac{1}{t_{1} t}+\frac{1}{u_{1} u}\right)+\frac{16}{s}\left(\frac{B_{1}(t)(2 s+u)}{t_{1}^{2}}+\frac{B_{1}(u)(2 s+t)}{u_{1}^{2}}\right)\right. \\
& +32 m^{2}\left(\frac{C_{1}(t)}{t t_{1}}+\frac{C_{1}(u)}{u u_{1}}\right)-\frac{16 m^{2}}{s t u}\left(s_{1} C_{1}(s)+(u-s) C_{1}(t)+(t-s) C_{1}(u)\right) \\
& +\frac{8 m^{2}}{s t u}\left(s t D_{0}(s, t)+s u D_{0}(s, u)-t u D_{0}(t, u)\right) \\
& \left.-\frac{16 m^{2}}{s} D_{0}(t, u)+\frac{8}{s^{2}} E_{0}(t, u)\right\} .
\end{aligned}
$$

and

$$
\begin{gathered}
\mathcal{M}_{++-}^{\tilde{t}_{i}}=\left(g_{h \tilde{t}_{i} \tilde{t}_{i}} v \Delta\right) \times\left\{-\frac{16 m_{H}^{2}}{s t u}+\frac{16 m^{2}}{s t u}\left(s_{1} C_{1}(s)+t_{1} C_{1}(t)+u_{1} C_{1}(u)\right)\right. \\
\left.-\frac{8 m^{2}}{s t u}\left(s t D_{0}(s, t)+s u D_{0}(s, u)+t u D_{0}(t, u)\right)\right\} .
\end{gathered}
$$

In these expressions we define

$$
s_{1} \equiv s-m_{H}^{2}, \quad t_{1} \equiv t-m_{H}^{2}, \quad u_{1} \equiv u-m_{H}^{2}, \quad \Delta=\sqrt{\frac{s t u}{8}} .
$$

The functions $B_{1}, C_{1}, D_{0}$ are 1-loop basic scalar integrals. They are functions of $s, t, u$, the mass of the particle in the loop, and the Higgs mass; their definitions can be found in [46]. The function $E_{0}$ introduced in [45] is an auxiliary function defined as

$$
E_{0}(s, t)=s C_{0}(s)+t C_{0}(t)+s_{1} C_{1}(s)+t_{1} C_{1}(t)-s t D_{0}(s, t),
$$

where $C_{0}$ is again a 1-loop scalar integral defined in [46].

The other $p p \rightarrow h j$ subprocesses $(q \bar{q} \rightarrow h g, q g \rightarrow h q, \bar{q} g \rightarrow h \bar{q})$ are controlled by a third function, the un-averaged amplitude squared

$$
\sum\left|M_{q \bar{q} \rightarrow H g}\right|^{2}(s, t, u)=\frac{16 \alpha_{s}^{3}}{\pi v^{2} s} \frac{t^{2}+u^{2}}{s_{1}^{2}}\left|\sum_{i=t, b, \tilde{t}_{1}, \tilde{t}_{2}} \mathcal{M}^{i}(q \bar{q} \rightarrow h g)\right|^{2} .
$$


The SM amplitudes can be found are in ref. [45], while for scalars running in the loop we have:

$$
\mathcal{M}^{\tilde{t}_{i}}(q \bar{q} \rightarrow h g)=-\left(g_{h \tilde{t}_{i} \tilde{t}_{i}} v\right) \times\left(\frac{1}{2}+m^{2} C_{1}(s)+\frac{s}{2 s_{1}} B_{1}(s)\right) .
$$

We can get the amplitudes for the subprocesses $q g \rightarrow h q$ and $g q \rightarrow h q$ from the above by swapping the Mandelstam variable $s$ and $u$, and $s$ and $t$ respectively.

Before we investigate the numerical impact of these corrections ${ }^{3}$ to the $p_{T}$-spectrum of a Higgs boson recoiling against one jet, let us discuss the qualitative feature of the above amplitudes. For the sake of illustration, we consider only $\mathcal{M}_{+++}$, but similar results hold for other amplitudes as well, and can be obtained by using the formulae presented in appendix A. We are particularly interested in how the SM (found in eq. (A.15) of ref. [45]) and top squark contributions behave when the momentum flowing through the loop is either much smaller or much larger than the loop particle masses. We denote $m$ as the scale of the superpartner masses, $m \sim m_{\tilde{t}_{1,2}}$, and distinguish two regimes:

- Low- $p_{T}$ limit $p_{T} \ll m$. Using the results in eq. (A.2), we find that the scalar contribution reduces to

$$
\left.\mathcal{M}_{+++}^{\tilde{t}_{i}}\right|_{p_{T} \ll m} \simeq-\frac{4}{3} \frac{\Delta}{p_{T}^{2}} \frac{g_{h \tilde{t}_{i} \tilde{t}_{i}} v}{m^{2}} \sim p_{T} \frac{m_{t}^{2}}{m^{2}}
$$

where we have used the definition of $\Delta$ [45], eq. (2.20), and the fact that $\Delta \sim p_{T}^{3}$. The factor $1 / \mathrm{m}^{2}$ originates from triangle loops. Finally, we have used the fact that the leading soft SUSY breaking part of the coupling $g_{h \tilde{t}_{i} \tilde{t}_{i}}$ is proportional to $m_{t}^{2} / v$, see eq. (2.3). In the SM case, the triangle loops contribute a factor of $1 / m_{t}^{2}$ but have two extra powers of $m_{t}$ in the numerator - one from the Yukawa coupling of the top, the other from a helicity flip imposed by the interaction with the Higgs. As a result:

$$
\left.\mathcal{M}_{+++}^{t}\right|_{p_{T} \ll m_{t}} \simeq-\frac{32}{3} \frac{\Delta}{p_{T}^{2}} \sim p_{T}
$$

with no dependence on the top mass, as in the total cross section $g g \rightarrow h$. Note that this low $p_{T}$ limit holds with a very good approximation also in the region $p_{T} \sim m, m_{t}$.

- High- $p_{T}$ limit $p_{T} \gg m, m_{t}, m_{H}$. In this limit, the amplitudes for both squarks and fermion loops reduce to single and double logarithms of $p_{T} / m$,

$$
\begin{aligned}
\left.\mathcal{M}_{+++}^{t}\right|_{p_{T} \gg m_{t}} & \simeq \frac{m_{t}^{2}}{p_{T}}\left(A_{0}+A_{1} \ln \left(\frac{p_{T}^{2}}{m_{t}^{2}}\right)+A_{2} \ln ^{2}\left(\frac{p_{T}^{2}}{m_{t}^{2}}\right)\right), \\
\left.\mathcal{M}_{+++}^{\tilde{t}_{i}}\right|_{p_{T} \gg m} & \simeq \frac{g_{h \tilde{t}_{i} \tilde{t}_{i}} v}{p_{T}}\left(\tilde{A}_{0}+\tilde{A}_{1} \ln \left(\frac{p_{T}^{2}}{m^{2}}\right)+\tilde{A}_{2} \ln ^{2}\left(\frac{p_{T}^{2}}{m^{2}}\right)\right),
\end{aligned}
$$

\footnotetext{
${ }^{3}$ In our calculations we will assume the only scalars running in the loop are $\tilde{t}_{1,2}$. If $\tan \beta$ is large, the bottom squark loops may be non-negligible. One could account them by adding the appropriate terms to the sums in equations (2.17) and (2.22). Additionally, there are extra diagrams that must be included coming from loops involving gluinos. When gluinos decouple, their effect gets absorbed into a correction of relative order $\alpha_{s}$ to the couplings of quarks and squarks to the Higgs. We neglect such contribution in our calculation of the above matrix elements, since these effects are of the same order as unknown higher-order QCD corrections [47].
} 
where $A_{i}, \tilde{A}_{i}(i=0,1,2)$ do not depend on the mass of the particle in the loops, but only on kinematic invariants. Also, $A_{i}$ are the same for all processes involving any fermion in the loops coupling in the same way a top does (e.g. a top partner), while $\tilde{A}_{i}$ are the same for all process with scalars in the loops.

Having seen the behavior of different components of the Higgs plus jet amplitude in the low and high- $p_{T}$ regime, we can combine things to get a sense of the behavior of the amplitude as a whole. In the limit where the $p_{T}$ of the Higgs is much less than $m_{t}$ or either of the top squark masses, the combination of eq. (2.25), (2.24) and (2.15) yields:

$$
\left.\mathcal{M}_{+++}\right|_{p_{T} \ll m_{t}, m_{\tilde{t}_{1}}, m_{\tilde{t}_{2}}} \simeq-\frac{32}{3} \frac{\Delta}{p_{T}^{2}}-\frac{4}{3} \frac{\Delta}{p_{T}^{2}} \sum_{i=\tilde{t}_{1}, \tilde{t}_{2}} \frac{g_{h \tilde{t}_{i} \tilde{t}_{i}} v}{m_{\tilde{t}_{i}}^{2}} \simeq 8 \frac{\Delta}{p_{T}^{2}}\left(-\frac{4}{3}+F_{g}\left(m_{\tilde{t}_{1}}, m_{\tilde{t}_{2}}, \theta_{\tilde{t}}\right)\right) .
$$

In this limit, the top squark contributions are combined into $F_{g}$, the same function appearing in inclusive production. In fact, in the $m_{t} \rightarrow \infty, m_{\tilde{t}_{1}}, m_{\tilde{t}_{2}} \gg m_{t}$ limit,

$$
\kappa_{g} \rightarrow\left(1-\frac{3}{4} F_{g}\right)
$$

the exact combination appearing in eq. (2.27). As such, the pattern of deviations in the low- $p_{T}$ regime of $h+$ jet production will mirror those of $p p \rightarrow h$. In particular, the parameter regions where the two stops cancel (e.g. when $F_{g}\left(m_{\tilde{t}_{1}}, m_{\tilde{t}_{2}}, \theta_{\tilde{t}}\right) \sim 0$ ), both inclusive $p p \rightarrow h$ and $p p \rightarrow h+$ jet processes will appear SM-like.

At intermediate $p_{T}, m_{t}<p_{T}<m_{\tilde{t}_{i}}$ we can approximate the amplitude as the sum of a high- $p_{T}$ piece (eq. (2.26a)) for the top plus a decoupled piece (eq. (2.24)) for the top squarks:

$$
\left.\mathcal{M}_{+++}\right|_{m_{t} \ll p_{T} \ll m_{\tilde{t}_{1}}, m_{\tilde{t}_{2}}} \frac{m_{t}^{2}}{p_{T}}\left(A_{0}+A_{1} \ln \left(\frac{p_{T}^{2}}{m_{t}^{2}}\right)+A_{2} \ln ^{2}\left(\frac{p_{T}^{2}}{m_{t}^{2}}\right)\right)+8 \frac{\Delta}{p_{T}^{2}} F_{g} .
$$

The contribution of the top quarks is again proportional to $F_{g}$ - so if there is a cancellation between two top squark contributions in the total cross section it will persist in this regime.

To break the cancellation, we must go to $p_{T}$ higher than the mass of the lighter stop. Here, we can approximate the amplitude as a high- $p_{T}$ contribution from the top loop and lightest top squark loop, and a decoupled piece for the heavier top squark:

$$
\begin{aligned}
\left.\mathcal{M}_{+++}\right|_{m_{t}, m_{\tilde{t}_{1}} \ll p_{T} \ll m_{\tilde{t}_{2}}} \simeq & \frac{m_{t}^{2}}{p_{T}}\left(A_{0}+A_{1} \ln \left(\frac{p_{T}^{2}}{m_{t}^{2}}\right)+A_{2} \ln ^{2}\left(\frac{p_{T}^{2}}{m_{t}^{2}}\right)\right) \\
& +\frac{g_{h \tilde{t}_{t_{i}}} v}{p_{T}}\left(\tilde{A}_{0}+\tilde{A}_{1} \ln \left(\frac{p_{T}^{2}}{m_{\tilde{t}_{1}}^{2}}\right)+\tilde{A}_{2} \ln ^{2}\left(\frac{p_{T}^{2}}{m_{\tilde{t}_{1}}^{2}}\right)\right) \\
& -\frac{4}{3} \frac{\Delta}{p_{T}^{2}} \frac{g_{h \tilde{t}_{i} \tilde{t}_{2}} v}{m_{\tilde{t}_{2}}^{\tilde{t}_{2}}} .
\end{aligned}
$$

The couplings $g_{h \tilde{t}_{i} \tilde{t}_{i}}$ multiply different kinematic functions rather than combining into $F_{g}$, so the amplitude is sensitive to the top squarks even when parameters conspire to make $F_{g} \sim 0$. Note that any cancellation between the contributions of the stops is also broken at very high $p_{T}$, i.e. $p_{T} \gg m_{\tilde{t}_{1}}, m_{\tilde{t}_{2}}$. 
To summarise, we have shown that by looking at the high- $p_{T}$ behavior of $h+\mathrm{j}$ once can break model degeneracies in the top squark sector, opening up sensitivity to parameter space that more inclusive searches cannot probe. However, the added information in $h+\mathrm{j}$ comes only when we consider $p_{T}$ higher than the mass of the lightest top squark. Whether or not one can expect to reach such kinematic $p_{T}$ regime at the LHC depends on the physical masses $m_{\tilde{t}_{1,2}}$. To get a more quantitative idea of the size of the deviation top squarks can cause, we turn to numerics.

\section{Numerical results}

Using the squared matrix elements in eqs. (2.17) and (2.22), we compute the $p_{T}$ spectrum of the Higgs $d \sigma / d p_{T}$. The actual calculation of the $p_{T}$ spectra results from interfacing a modified version of HERWIG [48] with the parton density toolkit HOPPET [49-57]. Our results have been validated against the existing program SusHi [20-22], using the MSSM input card, with very large masses for the bottom squarks and the gluinos. The main difference between our implementation and SusHi is that we compute the full $p_{T}$ spectrum with a Gaussian integrator in a single run, whereas SusHi uses a Monte-Carlo integrator to provide a single $p_{T}$-bin for each run. In terms of performance, with a single current CPU, with our implementation one can obtain the entire $p_{T}$-spectrum for a mass point in less than a second, whereas to run SusHi in one single $p_{T}$-bin would take about a minute.

In figure 1 , we show numerical results for $d \sigma / d p_{T}$ for $m_{\tilde{t}_{1}}=600 \mathrm{GeV}$ and four different values of the mass difference $\Delta m$, obtained with the MSTW2008NLO parton distribution set [58], for $\tan \beta=10$ and maximal mixing, i.e. $\theta=\pi / 4$, as well as the corresponding prediction in the SM. All distributions have been obtained by setting both renormalisation scale $\mu_{R}$ and factorisation scale $\mu_{F}$ equal to $\left(p_{T}+\sqrt{p_{T}^{2}+m_{H}^{2}}\right) / 2$. We note immediately that, for the chosen parameters, the difference between the SM spectrum and that with additional top squarks in the loops is not huge, at most $30 \%$ in the highest $p_{T}$ bins. The smallness of the effect is expected from the analytical results in section 2.2, eqs. (2.24)-(2.29).

We can compare these results with the contributions of fermionic top-partners to the same process discussed in ref. [30], in particular the high- $p_{T}$ behavior described in eq. (4.3) of that paper, where the dependence on $m$, the scale of new physics, goes as $m^{2} / p_{T}$, instead of $m_{t}^{2} / p_{T}$. Therefore, one would typically expect more sizeable effects from new fermionic top-partners than from stops.

In order to better assess deviations from the SM behaviour in the spectra, we construct the cumulative distribution $\sigma\left(p_{T}^{\text {cut }}\right)$, defined by

$$
\sigma\left(p_{T}^{\text {cut }}\right)=\int_{p_{T}^{\text {cut }}}^{\infty} d p_{T} \frac{d \sigma}{d p_{T}}
$$

and we consider the deviation $\delta\left(p_{T}^{\text {cut }}\right)$ of the cumulative cross section $\sigma\left(p_{T}^{\text {cut }}\right)$ from its expected value in the Standard Model as follows:

$$
\delta\left(p_{T}^{\text {cut }}\right)=\frac{\sigma\left(p_{T}^{\text {cut }}\right)-\sigma^{\mathrm{SM}}\left(p_{T}^{\text {cut }}\right)}{\sigma^{\mathrm{SM}}\left(p_{T}^{\text {cut }}\right)} .
$$




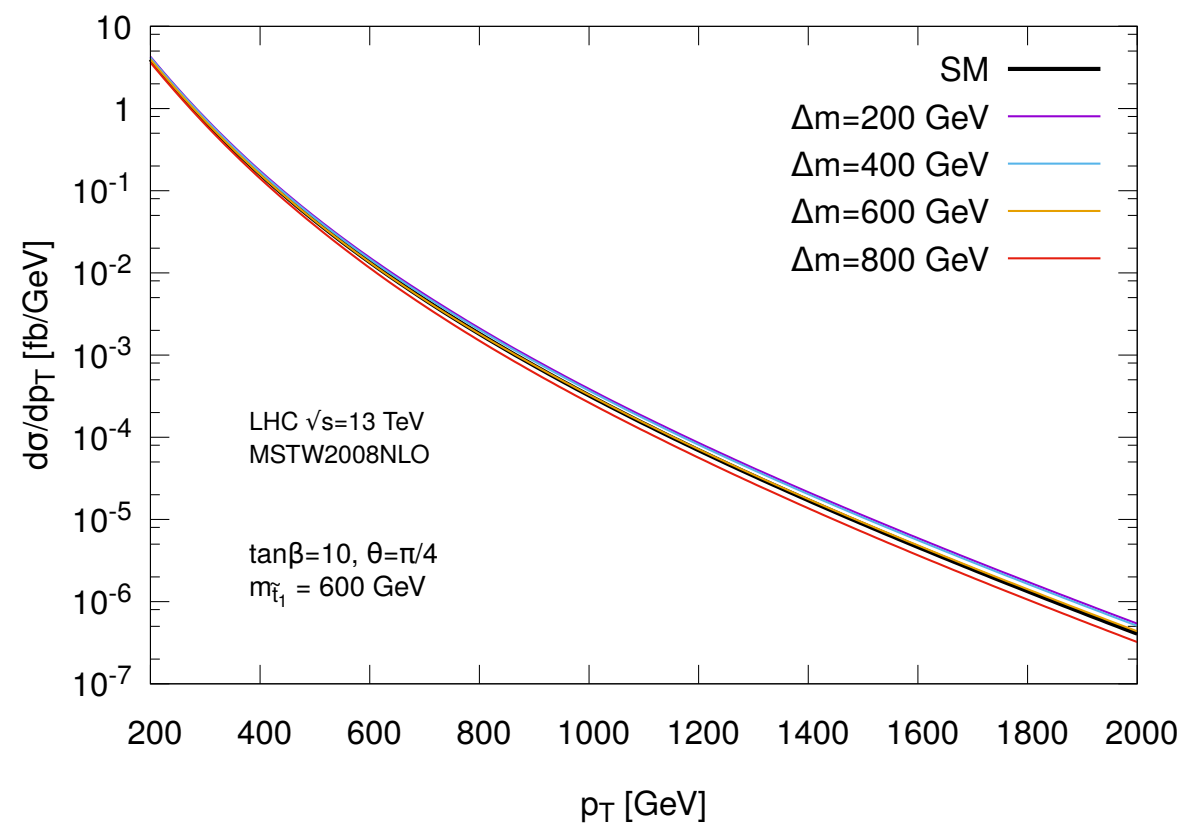

Figure 1. Transverse momentum spectra of the Higgs boson in the Standard Model, and for four different values of the mass difference $\Delta m$. See the main text for details.

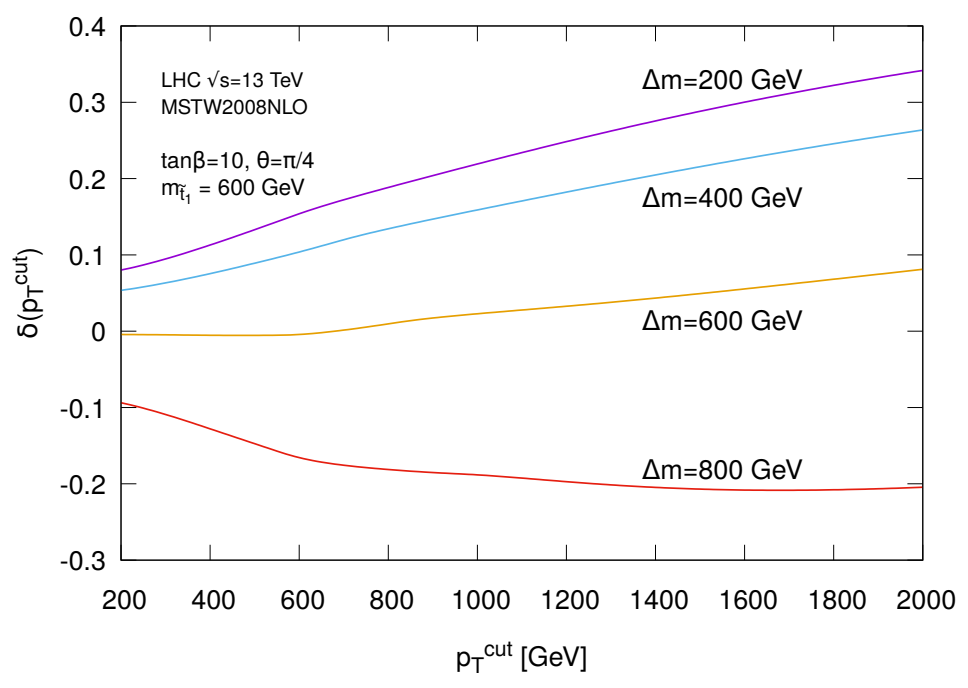

Figure 2. The deviation from SM expectation defined in eq. (3.2) as a function of $p_{T}^{\text {cut }}$ for $m_{\tilde{t}_{1}}=$ $600 \mathrm{GeV}$ and four different values of the mass difference $\Delta m$. See the main text for details.

The $\delta$ values corresponding to the parameters used in figure 1 are shown in figure 2 . Note that each prediction consists only of a single curve obtained by fixing $\mu_{R}=\mu_{F}=$ $\left(p_{T}+\sqrt{p_{T}^{2}+m_{H}^{2}}\right) / 2$ in each $p_{T}$ spectrum. A similar plot for the case of degenerate stops $(\Delta m=0)$ and various values of $m_{\tilde{t}_{1}}$ is shown in figure 3 . From this picture it is easier to see the effect of increasing the transverse momentum $p_{T}^{\text {cut }}$. In fact, the larger deviations are seen for $m_{\tilde{t}_{1}}=400 \mathrm{GeV}$, and are decreasing with increasing mass of the stops, in accordance with the arguments presented in the previous section. 


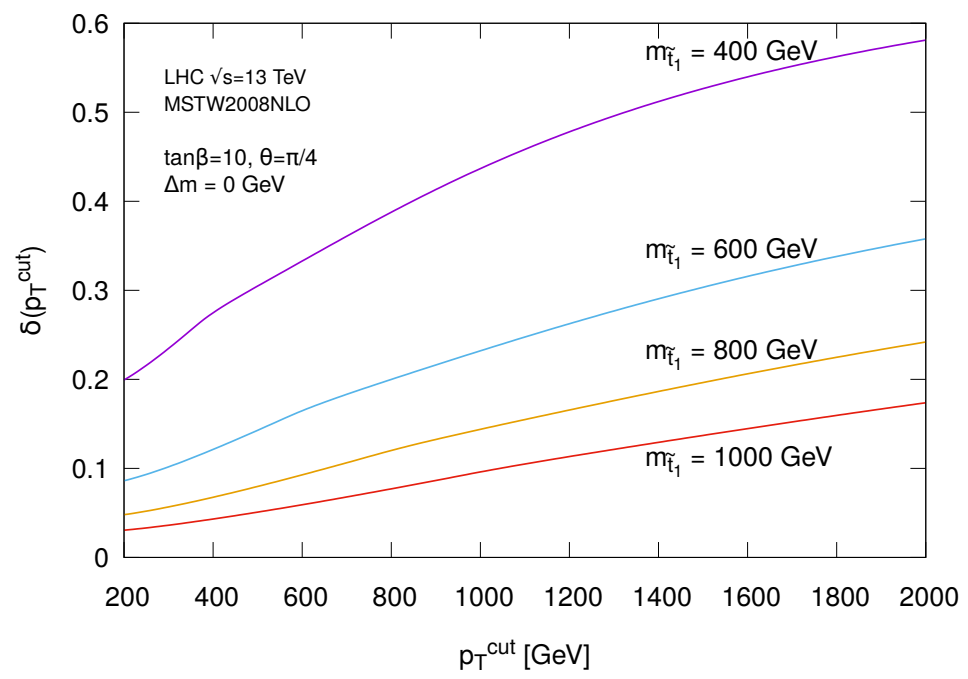

Figure 3. The deviation from SM expectation defined in eq. (3.2) as a function of $p_{T}^{\text {cut }}$ for $\Delta m=0$ (degenerate stops) and for different values of the stop masses. See the main text for details.
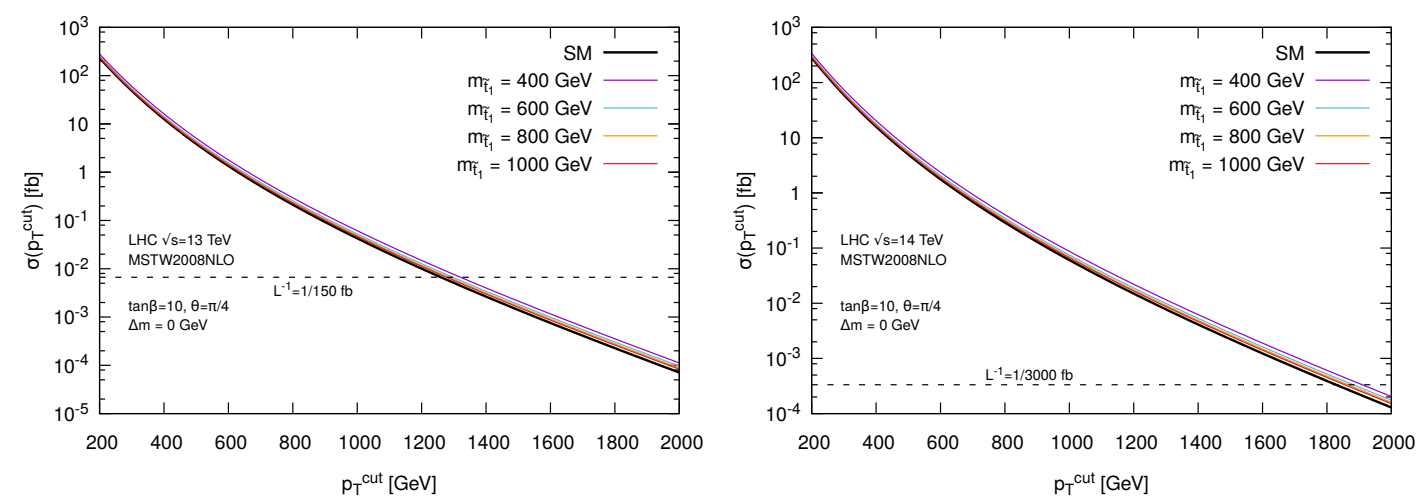

Figure 4. The integrated cross section $\sigma\left(p_{T}^{\text {cut }}\right)$ defined in eq. (3.2) as a function of $p_{T}^{\text {cut }}$ in the Standard Model, at the LHC with $\sqrt{s}=13 \mathrm{TeV}$ (left) and $\sqrt{s}=14 \mathrm{TeV}$ (right), and for four different values of the stop masses in the degenerate case $\Delta m=0$. See the main text for details.

In order to appreciate whether such deviations could be seen at the LHC, in figure 4 we plot the integrated cross section $\sigma\left(p_{T}^{\text {cut }}\right)$ of eq. (3.1) for the same choice of parameters as figure 3, for two different centre-of-mass energies corresponding to the present LHC setup (left) and the hight-luminosity setup. In each plot we see also a horizontal line, corresponding to the inverse of the maximum integrated luminosity $L$ available at the end of the runs of each setup ( $L=150 \mathrm{fb}^{-1}$ at the end of LHC Run 2 and $L=3000 \mathrm{fb}^{-1}$ at the end of the end of the High Luminosiy LHC (HL-LHC) programme). The crossing points with the integrated spectra are the values of $p_{T}^{\text {cut }}$ for which one event is expected, in case of perfect Higgs tagging in any decay mode. From the plots one could argue that, at the end of LHC Run 2, one could reasonably access transverse momenta up to about $1 \mathrm{TeV}$, and the reach moves up to around $1.5 \mathrm{TeV}$ at the end of the HL-LHC phase.

The above results are obtained by measuring the transverse momentum of either the Higgs or a jet recoiling with the Higgs. If we trigger on the jet, it might be interesting 

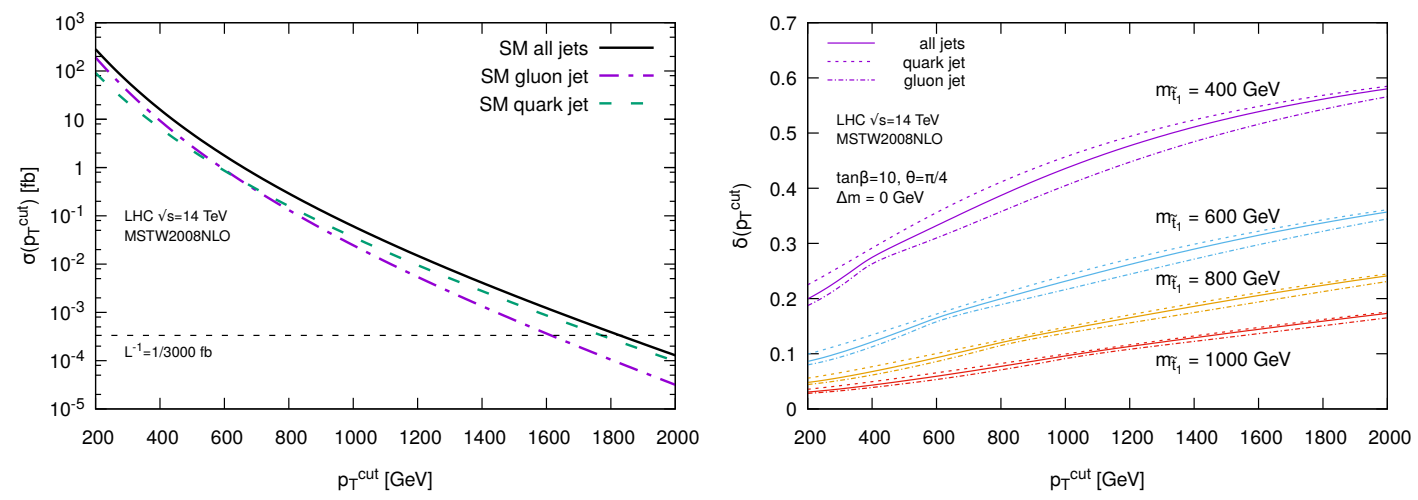

Figure 5. The integrated cross section $\sigma\left(p_{T}^{\text {cut }}\right)$ for the $\mathrm{SM}$ at $\sqrt{s}=14 \mathrm{TeV}$ (left) and the deviation from SM expectation defined in eq. (3.2) as a function of $p_{T}^{\text {cut }}$ for $\Delta m=0$ (degenerate stops) and for different values of the stop masses. See the main text for details.

to investigate whether the discrimination between quark and gluon jets, which has been the subject of several studies in recent years [59-65], might improve the sensitivity of the analysis we propose. Therefore, in the left panel of figure 5 we plot the integrated cross section $\sigma\left(p_{T}^{\text {cut }}\right)$ at the HL-LHC for the SM in the case in which we do not distinguish between quark and gluon jets (all jets), and two more curves corresponding to the case in which we are able to tag quark and gluon jets with $100 \%$ efficiency. We see that gluon jets dominate at low $p_{T}^{\text {cut }}$, whereas at high $p_{T}^{\text {cut }}$ the cross section is dominated by quark jets. In the right panel we then plot $\delta\left(p_{T}^{\text {cut }}\right)$ for the most sensitive scenario, that of degenerate stops with difference masses at HL-LHC, both when we cannot distinguish between quark and gluon jets, and when we can tag them with $100 \%$ efficiency. We observe that quark-gluon jet discrimination gives a mild increase in the sensitivity. We note also that the values $\delta\left(p_{T}^{\text {cut }}\right)$ are almost identical at $\sqrt{s}=13 \mathrm{TeV}$ and $14 \mathrm{TeV}$. This is why in the next section, which considers values of $p_{T}^{\text {cut }}$ up to $600 \mathrm{GeV}$, which should be within the reach of LHC Run 2, we restrict ourselves to the case $\sqrt{s}=13 \mathrm{TeV}$.

Last, we comment on the robustness of the variable $\delta$ with respect to higher order QCD corrections. Since a NLO calculation of the stop contribution has not been performed yet, the considerations below have the status of speculations. We wish nevertheless to point out how higher order corrections can affect $\delta$ under some assumptions based on existing SM results. Recently, a complete calculation of the differential Higgs+jet process in the SM at NLO with full top-mass effects has been performed [66]. The authors in ref. [66] find that the K-factor (the ratio $\mathrm{NLO} / \mathrm{LO}$ ) for this process is roughly 2 and quite flat with $p_{T}$. Hence, one could simply re-scale the SM contribution in eq. (3.2) by this $\mathrm{K}$ factor, improving the SM prediction to NLO. But the equivalent calculation for the BSM contribution is not known. Nevertheless, one can expect that the K-factor of the BSM contribution will be of similar size as the SM one. In fact, on one hand, one can argue that for large stop masses, the BSM contributions behaves in a similar way as the heavytop effective theory $\left(m_{t} \rightarrow \infty\right)$, which has a K-factor of about 2. On the other hand, ref. [66] also shows that for finite masses the K-factor one obtains is of the same order as that of heavy-top effective theory (HEFT), and the same as for the Higgs total cross 
section, both in HEFT and with mass effects. Also, related studies in which the Higgs+jet calculation is performed using different approximations for quark-mass effects $[67,68]$ give a K-factor of the similar size. Therefore, it seems that such a K-factor arises independently of the details of the interactions that produce the Higgs. Since producing the Higgs via stop loops just amounts to having a different short-distance mechanism to produce the Higgs, it is reasonable to expect a similar K-factor (flat with $p_{T}$ and of order 2) in the stop contribution to $\sigma$, and hence our calculation of the quantity $\delta$ and LO should be similar to that at NLO. Associating uncertainties to $\delta$ is tricky, in that numerator and denominator are higly correlated, and hence many theoretical uncertainties will cancel. For instance, we have checked that, with our calculation, a simultaneous variation of $\mu_{R}$ and $\mu_{F}$ in the range $1 / 2 \leq \mu_{R} / \mu_{F} \leq 2$ in the numerator and denominator in eq. (3.2) has a negligible impact on $\delta\left(p_{T}^{\text {cut }}\right)$. Therefore, no band associated with such scale variations has been shown in figure 2. Assuming that the theoretical uncertainty on $\delta$ is of the same order of the scale variations associated with $\sigma\left(p_{T}^{\text {cut }}\right)$, from the results of ref. [66] one expects an uncertainty of around $20 \%$ at NLO. This makes it difficult to appreciate deviations from the SM for a considerable range of stop parameters, see next section for details. In fact, scale uncertainties for NNLO Higgs+jet production in HEFT are around 10\% [69, 70]. If we assume scale uncertainties to be of the same order with full mass dependence, we can expect the situation to slightly improve once NNLO corrections will be computed. From the above considerations, it seems that $\delta\left(p_{T}^{\text {cut }}\right)$ may be robust against higher-order contributions, although this statement will have to be validated when NLO corrections to the BSM $p_{T}$ spectrum will become available. However, the above caveats have be kept in mind when looking at the plots in the next section, which are obtained from a calculation of $\delta$ at LO.

\section{Comparing inclusive Higgs and high- $p_{T}$ Higgs sensitivities}

Having reviewed the top squark contributions to inclusive Higgs production and Higgs plus jet, we now compare the LHC sensitivity in the two modes. For our comparison, we will fix $\theta$ and $\tan \beta$, and plot our results as contours in the $m_{\tilde{t}_{1}}, \Delta m$ plane. For $\theta$, we pick two benchmarks, $\theta=0, \theta=\pi / 4$; these correspond to the extremes of no mixing and maximal mixing among the different top squarks - other choices of mixing angle would fall between the two.

We now discuss the range of parameters that can be probed through the variable $\delta$, by showing different contours for $\delta\left(p_{T}^{\text {cut }}\right)$ (translated into a percent deviation) as a function of the mass of the lightest top squark $m_{\tilde{t}_{1}}$ and the top squark mass difference $\Delta m$. The filled contours correspond to $\delta\left(p_{T}^{\text {cut }}\right)$, as defined in eq. (3.2), whereas the dashed lines represent analogous contours for the total cross section, obtained from eq. (2.10). All the results we show correspond to $\tan \beta=10$. We have checked that other values of $\tan \beta$ lead to similar results. Furthermore, all the parameters we have considered are not excluded by present data of the Higgs $p_{T}$ spectrum [71].

For inclusive Higgs production, each value of $\theta, m_{\tilde{t}_{1}}, \Delta m$ can be mapped to $\kappa_{g}$ following eq. (2.10) and (2.11). Via $\kappa_{g}$, each parameter point maps onto an inclusive Higgs cross 
section which can then be compared to LHC limits (both current and projected). The result is shown in dashed lines in figures 6 and 7 . Regions to the left of the purple dashed line (green dashed line) are excluded by LHC Run-I [72] (LHC Run-II [73]) data. The blue, red, and black dashed lines show future sensitivity, quoted in terms of the percent deviation in the inclusive cross section coming from the top squarks (for the future bounds, the region to the left is excluded). As the difference between the two frames in figures 6 and 7 applies only to the Higgs plus jet mode, the dashed lines are the same in both panels. When the two top squarks are highly mixed we can easily spot the parameter region where a cancellation occurs between them: for $\theta=\pi / 4$, the cancellation occurs along a line between $\left(m_{\tilde{t}_{1}}, \Delta m\right)=(200 \mathrm{GeV}, 400 \mathrm{GeV})$ and extending to $(1000 \mathrm{GeV}, 700 \mathrm{GeV})$. When $\theta=0$, there can be no cancellation between top squarks and the bounds in $\left(m_{\tilde{t}_{1}}, \Delta m\right)$ space look qualitatively different.

The inclusive Higgs production contours are overlayed on top of Higgs plus jet $\delta\left(p_{T}^{\text {cut }}\right)$ contours, with $\delta\left(p_{T}^{\text {cut }}\right)$ defined in eq. (3.2). In the left panel, we show $\delta(200 \mathrm{GeV})$ while the right panel we show $\delta(600 \mathrm{GeV})$; in both frames we show $\delta$ deviations of 5,10,15 and $20 \%$. Focusing first on the maximal mixing case (figure 6) and comparing the two panels, we can see the impact of the Higgs $p_{T}$ cut. For $p_{T}^{\text {cut }}=200 \mathrm{GeV}$, we have $p_{T}^{\text {cut }} \lesssim m_{\tilde{t}_{1}}$ so the the top squarks can be considered as decoupled in the bins of the Higgs $p_{T}$ spectrum that contribute most to $\delta\left(p_{T}^{\text {cut }}\right)$. As shown in eq. (2.29), the top squark contribution in this regime is proportional to the same term $F_{g}$ appearing in eq. (2.15). Therefore, whenever there is a cancellation in the total Higgs cross section, there will also be a cancellation in $\delta\left(p_{T}^{\text {cut }}\right)$. This cancellation can also be appreciated by looking at the curves in figure 2 . Picking $\Delta m=600 \mathrm{GeV}$ as an example top squark mass, we see that we need to increase $p_{T}^{\text {cut }}$ to more than $600 \mathrm{GeV}$ to see an appreciable deviation of $\delta\left(p_{T}^{\text {cut }}\right)$ from one. In fact, for $p_{T}^{\text {cut }}>600 \mathrm{GeV}$ we start to open at least the loop containing the lighter top squark. This slightly larger sensitivity then is reflected in the right panel of figure 6 , as the e.g. $\delta=10 \%$ contour cuts out more parameter space than the $10 \%$ contour for inclusive Higgs production (dashed blue) for $m_{\tilde{t}_{1}} \lesssim 400 \mathrm{GeV}$.

In figure 7 , we show the analogous plots for the case in which no mixing occurs, i.e. $\theta=$ 0 . As in the maximal mixing scenario, when $p_{T}^{\text {cut }}=200 \mathrm{GeV}$ both the Higgs cross section and the Higgs $p_{T}$ spectrum have very similar sensitivities. This picture changes when increasing $p_{T}^{\text {cut }}$ to $600 \mathrm{GeV}$, where the sensitivity of the Higgs $p_{T}$ spectrum is essentially doubled with respect to that of the total cross section. This increase in mass sensitivity as one increases the cut is expected from the analytical analysis and can be seen in figure 3.

As there is no possible cancellation among top squarks when $\theta=0, F_{g}<0$ and the amplitudes for $p p \rightarrow h$ and $p p \rightarrow h+$ jet are always increased by new physics. In this way, the $\theta=0$ case is the scalar analogue of the contribution of a fermionic partner of the top presented in [30]. However, the sensitivity of the $p_{T}$ spectrum to contributions from fermionic top partners found in [30] is much larger than the sensitivity to scalars we find here. This difference is due to the specific interplay between a top and a top-partner in composite Higgs models for different values of the Higgs transverse momentum. In the case of composite Higgs models, there is a cancellation between the top contribution and the top partner contribution which occurs whenever both states are decoupled. As 

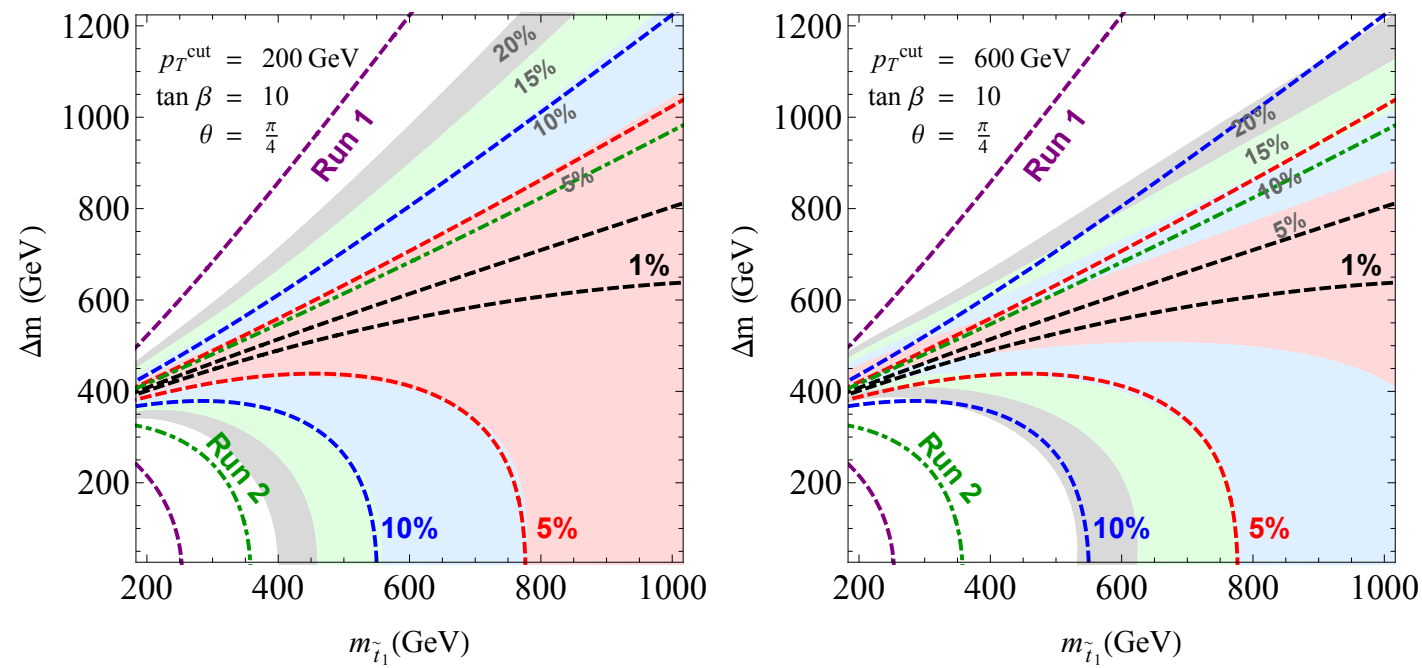

Figure 6. Contour plots for $\delta$ (as percent deviation) for the integrated Higgs $p_{T}$ spectrum (solid) and for the Higgs total cross section (dashed), as a function of the lighter top squark mass $m_{\tilde{t}_{1}}$ and of the top squark mass difference $\Delta m$, for $\tan \beta=10, \theta=\pi / 4$, and two different values of $p_{T}^{\text {cut }}$, namely $p_{T}^{\text {cut }}=200 \mathrm{GeV}$ and $p_{T}^{\text {cut }}=600 \mathrm{GeV}$.
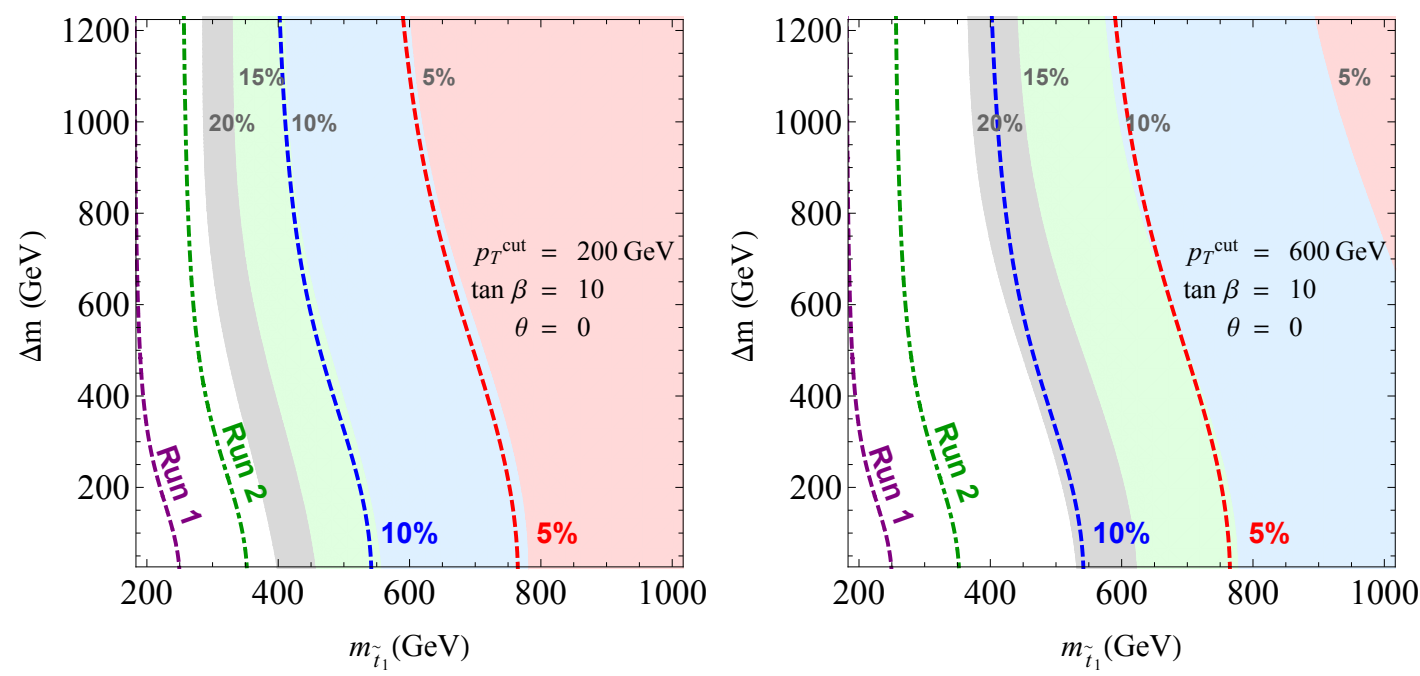

Figure 7. The same contour plots as in figure 6 , but for $\theta=0$.

the $p_{T}$ of the Higgs increases above $m_{t}$, a heavy top-partner stays decoupled, while the top quark behaves as a light particle, breaking the cancellation between contributions. This is not the case for top squarks, where there is a region of parameter space where the contribution of the two top squarks cancels when they are both decoupled, while the top quark contribution remains SM-like. Therefore, in order to break the cancellation, one needs to reach transverse momenta that exceed the mass of the lighter top squark. Furthermore, due to the fact that a top partner is a chiral fermion while a top squark is a scalar, the contribution to $p p \rightarrow h+$ jet from a heavy top squark is suppressed by one extra power of the top squark mass.

Finally, note that in this paper we are using Higgs data to indirectly probe top squarks. Currently, indirect top squark bounds are in the range of $m_{\tilde{t}} \sim 300 \mathrm{GeV}[41,44]$. The most 
recent direct searches for stops reach the $\mathrm{TeV}$ region, however this is not a fair comparison as direct searches are based on the assumption that stops decay into specific final states, largely involving missing energy signatures $[15,16]$.

\section{Discussion and outlook}

In this paper we studied how new colored scalars, top squarks, could affect the production of the Higgs boson. We were particularly interested in the interplay between inclusive gluon-fusion and differential Higgs production, $h+$ jet.

At first glance, one would think the inclusive production $g g \rightarrow h$ should be the dominant handle on possible new coloured particles. This production enjoys rich statistics and a solid experimental and theoretical understanding. The effect of new physics, however, could be reduced due to symmetries (such as in many models in Composite Higgs) or simply due to accidental cancellations (as in classes of SUSY sectors). Under these circumstances, the study of differential rates of the Higgs production in association with a high- $p_{T}$ jet is then the best handle to uncover new physics.

In prior work, ref. [30], we studied the sensitivity of the $h+$ jet channel in the quite dramatic case of fermionic top-partners in composite Higgs models, where low-energy theorems tend to protect the inclusive production from any variations from the SM; see also refs. [31, 32].

In this paper we have focused on scalar top-partners, and in particular on supersymmetric top squarks, where no such low-energy theorems are present. Nevertheless, we found that there is an interesting interplay between the information contained in the $h+$ jet differential production and the inclusive production. We presented analytical expressions for the corresponding amplitudes including the dependence on the stop spectrum, as opposed to previous studies [18-22].

Obviously, if an accidental cancellation occurs in the stop sector leading to a reduced gluon fusion rate [18], the differential production could become the best handle to discover new physics. Even if no dramatic cancellation occurs, the $h+$ jet rate still adds value to the search for new physics. Searches in gluon-fusion inclusive and the boosted Higgs topologies face very different background challenges. Indeed, as we have seen in searches by ATLAS and CMS, some decays of the Higgs may be more accessible in the boosted regime than in the inclusive case. Hence, a combined analysis of the two topologies would provide us with the best handle to dig top squarks from the LHC data.

We have obtained analytical expressions for the $h+$ jet amplitude in various $p_{T}$ regimes and found that the information one could gain on stops using differential rates is, unsurprisingly, concentrated in the regime $p_{T} \gtrsim m_{\tilde{t}}$. We then performed a numerical study to evaluate these relative effects. As a simple measure of the differential rate, we have defined a cumulative variable, namely the excess of events above a certain bin in $p_{T}$ with respect to the SM, $\delta\left(p_{T}\right)$. We argued that $\delta$ may be more robust against theoretical and experimental uncertainties than a fully differential study. With this naive measure of new physics in the high- $p_{T}$ region, we chose two benchmark values $p_{T}>200$ and $600 \mathrm{GeV}$ and compared future prospects for inclusive and differential information under some assumptions regarding the level of uncertainties for each topology. As expected, larger cuts on 
$p_{T}$ can lead to increased sensitivity, but the gain has to be weighted against the loss of statistics. This is a similar situation encountered when using the missing-energy distribution in searches for SUSY Dark Matter [74] and a similar detailed analysis should be done. Such an analysis in the case of Higgs + jet topologies is feasible (at LO) with current Monte-Carlo event generators, through aMCSusHi, the interface of the fixed-order program SusHi to aMC@NLO [23].

In order to completely assess which values of $\delta$ can be actually probed by experiments we need to be able to accurately determine the SM contribution. This requires considering all possible backgrounds to Higgs production in the selected decay channels, e.g. $h \rightarrow \gamma \gamma$ or to four-leptons, estimating the associated systematic uncertainties and performing a suitable statistical data analysis. Such a study is beyond the scope of this paper, but one can examine the results in ref. [75], where the authors considered the effect of $\kappa_{g}$ as an effective operator (see also ref. [76] for an NLO analysis). Specifically, using the transverse momentum spectrum of a Higgs decaying into $\tau \tau$ and $W W^{*}$, recoiling against a jet with $p_{t, j}>200 \mathrm{GeV}$, and $3 \mathrm{ab}^{-1}$ of luminosity at the HL-LHC, ref. [75] claims that it is possible to exclude at $95 \%$ confidence level values of $\kappa_{g}$ in the range $\kappa_{g}<-0.4$ and $\kappa_{g}>0.3$, with the additional constraint that no deviation is seen in the Higgs total cross section, and assuming that experimental systematic uncertainties are at most $10 \% .{ }^{4}$ For $p_{t, j}>200 \mathrm{GeV}$, these values of $\kappa_{g}$ correspond to roughly a $6-7 \%$ deviation from the SM. This means that obtaining a similar sensitivity in the present case is not unreasonable.

However, the analysis in ref. [75] relies on the dramatic growth with energy of the higher-dimensional operator, which in turns results in deviations from the SM on the Higgs transverse momentum spectrum which become as big as $80 \%$ for $p_{T}>600 \mathrm{GeV}$. This is quite different from our situation, where increasing the cut on the jet transverse momentum does not lead to huge deviations from the SM. Indeed, let us compare the prospects drawn in ref. [75] with a simple case where one single stop dominates the phenomenology. Namely, let us take eqs. (2.28) and (2.15) in the case $m_{\tilde{t}_{1}} \ll m_{\tilde{t}_{2}}$ and $\theta=0$, leading to $\kappa_{g} \simeq 1+\frac{4 m_{t}^{2}}{m_{\tilde{t}_{1}}^{2}}$. A $10 \%$ reach in $\kappa_{g}$ would then mean a limit $m_{\tilde{t}_{1}}>1 \mathrm{TeV}$, which is clearly beyond the sensitivity we expect when looking at the full stop contributions.

In conclusion, we hope this paper serves to motivate the experimental collaborations to perform a combined analysis of gluon-fusion and differential information to search for new physics. We have provided an analytical understanding of the differential rates in various regimes of $p_{T}$ and defined a useful, but rather simplistic, variable $\delta$ to encompass some of the differential information. We also hope to encourage theorists to perform the calculation of the differential distribution for stops at NLO QCD, which would be ultimately needed to sensibly compare with the SM predictions in the high- $p_{T}$ tails.

\section{Acknowledgments}

The work of AB and VS is supported by the Science Technology and Facilities Council (STFC) under grant number ST/P000819/1. The work of AM was partially supported by the National Science Foundation under Grant No. PHY-1520966.

\footnotetext{
${ }^{4}$ The prospects of measuring the coupling of the Higgs to gluons in future lepton colliders are promising. For example, $\Delta B R(h \rightarrow g g) / B R(h \rightarrow g g)=4 \%$ and $7 \%$ for ILC250, ILC500 [77].
} 


\section{A Higgs plus one jet for large top squark masses}

Here we give analytical expressions for the helicity amplitudes introduced in section 2.2 in the "decoupling" limit $m^{2} \gg m_{H}^{2}, s,|t|,|u|$, where $m$ is the scalar running in the loops.

First, we give the expansion of the scalar integrals appearing in the amplitudes:

$$
\begin{array}{rlrl}
B_{1}\left(q^{2}\right) & \simeq \frac{q^{2}-m_{H}^{2}}{6 m^{2}}, & C_{1}\left(q^{2}\right) \simeq-\frac{1}{2 m^{2}}-\frac{q^{2}+m_{H}^{2}}{24 m^{4}}, \\
D_{0}(s, t) & \simeq \frac{1}{6 m^{4}}, \quad E_{0}(s, t) \simeq \frac{u}{m^{2}} .
\end{array}
$$

This gives

$$
\begin{aligned}
& M_{+++}^{\tilde{t}_{i}} \simeq-\frac{\Delta g_{h \tilde{t}_{i} \tilde{t}_{i}} v}{s t u} \frac{4}{3} \frac{s^{2}}{m^{2}}, \quad M_{++-}^{\tilde{t}_{i}} \simeq \frac{\Delta g_{h \tilde{t}_{i} \tilde{t}_{i}} v}{s t u} \frac{4}{3} \frac{m_{H}^{4}}{m^{2}}, \\
& M_{-+-}^{\tilde{t}_{i}} \simeq-\frac{\Delta g_{h \tilde{t}_{i} \tilde{t}_{i}} v}{s t u} \frac{4}{3} \frac{t^{2}}{m^{2}}, \quad M_{-++}^{\tilde{t}_{i}} \simeq-\frac{\Delta g_{h \tilde{t}_{i} \tilde{t}_{i}} v}{s t u} \frac{4}{3} \frac{u^{2}}{m^{2}} .
\end{aligned}
$$

Similarly,

$$
\begin{aligned}
& \mathcal{M}^{\tilde{t}_{i}}(q \bar{q} \rightarrow h g) \simeq-\frac{g_{h \tilde{t}_{i} \tilde{t}_{i}} v}{24} \frac{s_{1}}{m^{2}}, \quad \quad \mathcal{M}^{\tilde{t}_{i}}(q g \rightarrow h q) \simeq-\frac{g_{h \tilde{t}_{i} \tilde{t}_{i}} v}{24} \frac{u_{1}}{m^{2}}, \\
& \mathcal{M}^{\tilde{t}_{i}}(g q \rightarrow h q) \simeq-\frac{g_{h \tilde{t}_{i} \tilde{t}_{i}} v}{24} \frac{t_{1}}{m^{2}} .
\end{aligned}
$$

\section{B Higgs plus one jet in the soft and collinear limit}

In this appendix we report the soft and collinear limits of the amplitudes and matrix elements for Higgs plus one-jet production computed in section 2.2. First, this information constitutes an important validation tool for our calculation. Also, since these checks may involve non-trivial cancellations between the contributions of different scalar one-loop integrals, we believe that the methods and the formulae reported here might be useful for similar studies aiming at exploiting the analytical properties of the matrix elements.

The main property of soft and collinear limits of matrix elements is that they factorize into the product of the tree-level matrix element and universal functions. Therefore, we first need the expression of the Born matrix element. Due to conservation of angular momentum, the amplitude for the process $g g \rightarrow h$ is non-zero only if the two gluons have the same helicity, say both positive. The un-averaged matrix element squared for this process is

$$
\left|M_{g g \rightarrow h}\right|^{2}=\frac{\left(N_{c}^{2}-1\right) \alpha_{s}^{2}}{32 \pi^{2} v^{2}}\left|\sum_{i=t, b, \tilde{t}_{1}, \tilde{t}_{2}} \mathcal{M}_{++}^{i}\right|^{2}
$$

The top squark contribution to the above equation is

$$
\mathcal{M}_{++}^{\tilde{t}_{i}}=2 g_{h \tilde{t}_{i} \tilde{t}_{i}} v\left(1+2 m^{2} C_{0}\left(m_{H}^{2}\right)\right)
$$

with $g_{h \tilde{t}_{i} \tilde{t}_{i}}$ is either of the couplings defined in equations (2.2), (2.3). 


\section{B.1 Soft limit}

The soft limit $p_{3} \rightarrow 0$ corresponds to

$$
s \rightarrow m_{H}^{2}, \quad u, t \rightarrow 0, \quad s_{1} \rightarrow 0, \quad u_{1}, t_{1} \rightarrow-m_{H}^{2} .
$$

Keeping the most relevant terms in this limit, (2.18) gives

$$
\begin{aligned}
\frac{\mathcal{M}_{+++}^{\tilde{t}_{i}}}{g_{h \tilde{t}_{i} \tilde{t}_{i} v \Delta} v} & \frac{16}{t u}-\frac{16 m^{2}}{m_{H}^{2} t u}\left(t C_{0}(t)+u C_{0}(u)-2 m_{H}^{2} C_{0}\left(m_{H}^{2}\right)\right) \\
& -\frac{8 m^{2}}{m_{H}^{2} t u}\left(s t D_{0}(s, t)+s u D_{0}(s, u)-t u D_{0}(t, u)\right) .
\end{aligned}
$$

In the soft limit the relevant integral limits are

$$
t C_{0}(t) \rightarrow 0, \quad u C_{0}(u) \rightarrow 0, \quad s t D_{0}(s, t) \rightarrow 0, \quad u s D_{0}(u, s) \rightarrow 0, \quad u t D_{0}(u, t) \rightarrow 0,
$$

which gives

$$
\begin{aligned}
\mathcal{M}_{+++}^{\tilde{t}_{i}} & \simeq \frac{16}{t u} g_{h \tilde{t}_{i} \tilde{t}_{i}} v \Delta\left(1+2 m^{2} C_{0}\left(m_{H}^{2}\right)\right) \\
& \simeq(\sqrt{2})^{3} \sqrt{\frac{s}{t u}} \mathcal{M}_{++}^{\tilde{t}_{i}} .
\end{aligned}
$$

Similarly, the other helicity amplitude (2.19) becomes

$$
\begin{aligned}
\frac{\mathcal{M}_{++-}^{\tilde{t}_{i}}}{g_{h \tilde{t}_{i} \tilde{t}_{i}} v \Delta} \simeq & -\frac{16}{t u}+\frac{16 m^{2}}{m_{H}^{2} t u}\left(t C_{0}(t)+u C_{0}(u)-2 m_{H}^{2} C_{0}\left(m_{H}^{2}\right)\right) \\
& -\frac{8 m^{2}}{m_{H}^{2} t u}\left(s t D_{0}(s, t)+s u D_{0}(s, u)+t u D_{0}(t, u)\right) .
\end{aligned}
$$

Evaluating again all scalar integrals in the soft limit we get

$$
\begin{aligned}
\mathcal{M}_{++-}^{\tilde{t}_{i}} & \simeq-\frac{16}{t u} g_{h \tilde{t}_{i} \tilde{t}_{i}} v \Delta\left(1+2 m^{2} C_{0}\left(m_{H}^{2}\right)\right) \\
& \simeq-(\sqrt{2})^{3} \sqrt{\frac{s}{t u}} \mathcal{M}_{++}^{\tilde{t}_{i}} .
\end{aligned}
$$

These expressions have to be compared with the universal behavior of helicity amplitudes $[78,79]:^{5}$

$$
\begin{aligned}
\mathcal{M}_{+++}^{\tilde{t}_{i}} & =(\sqrt{2})^{3} \frac{\left\langle p_{1} p_{2}\right\rangle}{\left\langle p_{1} p_{3}\right\rangle\left\langle p_{3} p_{2}\right\rangle} \mathcal{M}_{++}^{\tilde{t}_{i}}, \\
\mathcal{M}_{++-}^{\tilde{t}_{i}} & =-(\sqrt{2})^{3} \frac{\left[p_{1} p_{2}\right]}{\left[p_{1} p_{3}\right]\left[p_{3} p_{2}\right]} \mathcal{M}_{++}^{\tilde{t}_{i}} .
\end{aligned}
$$

\footnotetext{
${ }^{5}$ The $\sqrt{2}$ factors comes from the differing normalisation factors for gauge group generators $\operatorname{tr}\left[T^{a} T^{b}\right]=\delta^{a b}$ in the spinor helicity formalism, compared to the usual $\operatorname{tr}\left[T^{a} T^{b}\right]=\frac{1}{2} \delta^{a b}$. This is compensated by a relative $\sqrt{2}$ factor associated to the gauge coupling.
} 
Since we have not used the spinor-helicity formalism, it is not immediate to rephrase our expressions in terms of helicity products. However, for real momenta, spinor products are simply equal to the square root of the relevant momentum invariant, up to a phase. The universal soft factor has an implicit helicity set by the helicity of the soft gluon, and so the choice of translating to angle or square bracket spinor products is fixed by this. We then obtain from (B.6) and (B.8) that $\mathcal{M}_{+++}^{\tilde{t}_{i}}$ and $\mathcal{M}_{++-}^{\tilde{t}_{i}}$ have the correct behavior (B.9) in the soft limit, modulo an overall phase that depends on the gluon helicity. This phase is the same as for the standard model case, and therefore can be factored out of each helicity amplitude and will not contribute to the amplitude squared.

\section{B.2 Collinear limits}

We consider the collinear limit $u \rightarrow 0$ where $p_{1}$ becomes collinear to $p_{3}$. Introducing the splitting fraction $z=\frac{m_{H}^{2}}{s}$, the invariants take the limiting values

$$
u \rightarrow 0, \quad s=\frac{m_{H}^{2}}{z}, \quad t \rightarrow-\frac{1-z}{z} m_{H}^{2}, \quad s_{1} \rightarrow-t, \quad t_{1} \rightarrow-s, \quad u_{1} \rightarrow-m_{H}^{2} .
$$

In this limit $u C_{0}(u) \rightarrow 0$, whereas $s C_{0}(s)$ and $t C_{0}(t)$ stay finite. For the box integrals, we have

$$
s t D_{0}(s, t) \rightarrow 2\left[s C_{0}(s)+t C_{0}(t)-m_{H}^{2} C_{0}\left(m_{H}^{2}\right)\right], \quad s u D_{0}(s, u) \rightarrow 0, \quad u t D_{0}(u, t) \rightarrow 0 .
$$

In this limit we get

$$
\begin{aligned}
\frac{\mathcal{M}_{+++}^{\tilde{t}_{i}}}{g_{h \tilde{t}_{i} \tilde{t}_{i}} v \Delta} \simeq & \frac{16}{t u}\left(1+\frac{t}{u_{1}}\right)+\frac{32 m^{2}}{u u_{1}^{2}} u_{1} C_{1}(u) \\
& -\frac{16 m^{2}}{s t u}\left[s_{1} C_{1}(s)-\frac{s}{t_{1}} t_{1} C_{1}(t)+\frac{t-s}{u_{1}} u_{1} C_{1}(u)\right] \\
& +\frac{8 m^{2}}{s t u}\left[s t D_{0}(s, t)+s u D_{0}(s, u)-t u D_{0}(t, u)\right] \\
\simeq & -\frac{16 z}{(1-z) u}\left(1+\frac{1-z}{z}\right)-\frac{32 m^{2}}{m_{H}^{4} u} m_{H}^{2} C_{0}\left(m_{H}^{2}\right) \\
& +\frac{16 m^{2} z^{2}}{(1-z) m_{H}^{4} u}\left[s C_{0}(s)-m_{H}^{2} C_{0}\left(m_{H}^{2}\right)+t C_{0}(t)\right. \\
& -\frac{16 m^{2} z^{2}}{(1-z) m_{H}^{4} u}\left[s C_{0}(s)+t C_{0}(t)-m_{H}^{2} C_{0}\left(m_{H}^{2}\right)\right] \\
= & -\frac{16}{(1-z) m_{H}^{2} u}\left[1+2 m^{2} C_{0}\left(m_{H}^{2}\right)\right] .
\end{aligned}
$$


Similarly, for the other helicity configuration we obtain

$$
\begin{aligned}
\frac{\mathcal{M}_{++-}^{\tilde{t}_{i}}}{g_{h \tilde{t}_{i} \tilde{t}_{i}} v \Delta} \simeq & -\frac{16 m_{H}^{2}}{s t u}\left\{1-\frac{m^{2}}{m_{H}^{2}}\left[s C_{0}(s)-m_{H}^{2} C_{0}\left(m_{H}^{2}\right)+t C_{0}(t)\right.\right. \\
& +\frac{m^{2}}{m_{H}^{2}}\left[s C_{0}(s)+t C_{0}(t)-m_{H}^{2} C_{0}\left(m_{H}^{2}\right)-m_{H}^{2} C_{0}\left(m_{H}^{2}\right)\right] \\
\simeq & \frac{16 z^{2}}{(1-z) m_{H}^{2} u}\left[1+2 m^{2} C_{0}\left(m_{H}^{2}\right)\right] .
\end{aligned}
$$

Now in the collinear case the limit depends on the helicity of each collinear leg. This means that there are two more possibilities to consider, and therefore we should additionally look at the limit of the two helicity amplitudes $\mathcal{M}_{-+-}^{\tilde{t}_{i}}$ and $\mathcal{M}_{-++}^{\tilde{t}_{i}}$. The first can simply be found by interchanging $s$ and $t$ in $\mathcal{M}_{+++}^{\tilde{t}_{i}}$. As this does not affect the relevance of terms in this limit, the switch can be effected by making the substitution $z \rightarrow \frac{z}{z-1}$ in the limit form, and so from (B.12) we have

$$
\begin{aligned}
\frac{\mathcal{M}_{-+-}^{\tilde{t}_{i}}}{g_{h \tilde{t}_{i} \tilde{t}_{i}} v \Delta} & \simeq-\frac{16}{\left(1-\frac{z}{z-1}\right) m_{H}^{2} u}\left[1+2 m^{2} C_{0}\left(m_{H}^{2}\right)\right] \\
& \simeq-\frac{16}{m_{H}^{2} u}(1-z)\left[1+2 m^{2} C_{0}\left(m_{H}^{2}\right)\right] .
\end{aligned}
$$

Extracting the collinear limit from $\mathcal{M}_{-++}^{\tilde{t}_{i}}$ is trickier. It is obtained from $\mathcal{M}_{+++}^{\tilde{t}_{i}}$ by exchanging $s$ and $u$, and as such the relevant terms in the collinear limit will be different in structure from the above cases. One has

$$
\begin{aligned}
\frac{\mathcal{M}_{-++}^{\tilde{t}_{i}}(s, t, u)}{g_{h \tilde{t}_{i} \tilde{t}_{i}} v \Delta}= & \frac{\mathcal{M}_{+++}^{\tilde{t}_{i}}}{g_{h \tilde{t}_{i} \tilde{t}_{i}} v \Delta} \\
\simeq & \frac{16}{u}\left(\frac{t}{s_{1}^{2}} B_{1}(s)+\frac{s}{t_{1}^{2}} B_{1}(t)\right) \\
& -\frac{16 m^{2}}{s t u}\left(u_{1} C_{1}(u)+\frac{s}{t_{1}} t_{1} C_{1}(t)+\frac{t}{s_{1}} s_{1} C_{1}(s)\right) \\
& +\frac{8 m^{2}}{s t u}\left(u t D_{0}(u, t)+u s D_{0}(u, s)-s t D_{0}(s, t)\right) \\
& -\frac{16 m^{2}}{s t u} s t D_{0}(s, t)+\frac{8}{u^{2}} E_{0}(s, t) .
\end{aligned}
$$

Notice that, since the term containing $E_{0}(s, t)$ is proportional to $1 / u^{2}$, one needs to keep the linear terms in $u$ in the small- $u$ expansion of $E_{0}(s, t)$. In particular, as $E_{0}(s, t)$ is the linear combination defined in (2.21) one cannot use the limit of eq. (B.11) to evaluate $s t D_{0}(s, t)$, but rather one must use the extended version

$$
\begin{aligned}
s t D_{0}(s, t) \rightarrow & 2\left(1-\frac{2 m^{2} u}{s t}\right)\left[s C_{0}(s)+t C_{0}(t)-m_{H}^{2} C_{0}\left(m_{H}^{2}\right)\right] \\
& +\frac{2 u}{s t}\left[s B_{0}(s)+t B_{0}(t)-m_{H}^{2} B_{0}\left(m_{H}^{2}\right)\right] .
\end{aligned}
$$

Substituting this expression in (B.15) leads to $\mathcal{M}_{-++} \simeq 0$ in the collinear limit $u \rightarrow 0$. 
Collecting all results we have

$$
\begin{aligned}
& \mathcal{M}_{+++}^{\tilde{t}_{i}} \simeq \frac{-(\sqrt{2})^{3}}{z \sqrt{(1-z)} \sqrt{-u}} \mathcal{M}_{++}^{\tilde{t}_{i}}, \\
& \mathcal{M}_{++-}^{\tilde{t}_{i}} \simeq \frac{z(\sqrt{2})^{3}}{\sqrt{(1-z)} \sqrt{-u}} \mathcal{M}_{++}^{\tilde{t}_{i}}, \\
& \mathcal{M}_{-+-}^{\tilde{t}_{i}} \simeq \frac{-(1-z)^{2}(\sqrt{2})^{3}}{z \sqrt{(1-z)} \sqrt{-u}} \mathcal{M}_{++}^{\tilde{t}_{i}}, \\
& \mathcal{M}_{-++}^{\tilde{t}_{i}} \simeq 0 .
\end{aligned}
$$

To check the correctness of the above limits, we have to translate our conventions for helicity and splitting fraction into the ones available in the literature, in which all momenta are considered to be outgoing. First, we need to swap the helicity of each incoming particle. Additionally, the relation of $z$ to the momenta is different when the collinear gluons are outgoing. One can switch between the two cases by making the replacement $z \rightarrow \frac{1}{z}$. Adopting the usual convention of associating negative momentum signs to angle spinors we expect the behavior $[78,79]$

$$
\begin{aligned}
& \frac{\mathcal{M}_{+++}^{\tilde{t}_{i}}}{\mathcal{M}_{++}^{\tilde{t}_{i}}} \simeq \operatorname{Split}_{+}\left(-1^{-}, 3^{+} ; \frac{1}{z}\right)=\frac{-(\sqrt{2})^{3}}{z \sqrt{1-z}\left\langle p_{1} p_{3}\right\rangle}, \\
& \frac{\mathcal{M}_{++-}^{\tilde{t}_{i}}}{\mathcal{M}_{++}^{\tilde{t}_{i}}} \simeq \operatorname{Split}_{+}\left(-1^{-}, 3^{-} ; \frac{1}{z}\right)=\frac{z(\sqrt{2})^{3}}{\sqrt{1-z}\left[p_{1} p_{3}\right]}, \\
& \frac{\mathcal{M}_{-+-}^{\tilde{t}_{i}}}{\mathcal{M}_{++}^{\tilde{t}_{i}}} \simeq \operatorname{Split}_{+}\left(-1^{+}, 3^{-} ; \frac{1}{z}\right)=\frac{-(1-z)^{2}(\sqrt{2})^{3}}{z \sqrt{1-z}\left\langle p_{1} p_{3}\right\rangle}, \\
& \frac{\mathcal{M}_{-++}^{\tilde{t}_{i}}}{\mathcal{M}_{++}^{\tilde{t}_{i}}} \simeq \operatorname{Split}_{+}\left(-1^{+}, 3^{+} ; \frac{1}{z}\right)=0 .
\end{aligned}
$$

We must now translate (B.17) to helicity language. The translation from Mandelstam variables to spinor invariants is similar to the soft case, although the helicity consideration is slightly subtler. As the three legs of the splitting amplitude are collinear, we no longer have information about the contribution from each individual leg, as the helicity spinors become proportional. Instead what matters is the overall (outgoing) helicity of the three, which governs whether it is appropriate to translate to angle or square brackets, and with this consideration we indeed find the correct momentum dependence. However, this is not relevant in the end because, up to an overall phase $\left[p_{1} p_{3}\right] \sim\left\langle p_{1} p_{3}\right\rangle \sim \sqrt{-u}$.

Open Access. This article is distributed under the terms of the Creative Commons Attribution License (CC-BY 4.0), which permits any use, distribution and reproduction in any medium, provided the original author(s) and source are credited.

\section{References}

[1] K. Fujii et al., Physics case for the $250 \mathrm{GeV}$ stage of the international linear collider, arXiv: 1710.07621 [INSPIRE]. 
[2] TleP Design Study Working Group collaboration, M. Bicer et al., First look at the physics case of TLEP, JHEP 01 (2014) 164 [arXiv:1308.6176] [INSPIRE].

[3] J. Fan, M. Reece and L.-T. Wang, Precision natural SUSY at CEPC, FCC-ee and ILC, JHEP 08 (2015) 152 [arXiv: 1412.3107] [INSPIRE].

[4] S. Dawson et al., Working group report: Higgs boson, arXiv:1310.8361 [INSPIRE].

[5] CLIC Physics Working Group collaboration, E. Accomando et al., Physics at the CLIC multi-TeV linear collider, hep-ph/0412251 [INSPIRE].

[6] M.E. Peskin, Comparison of LHC and ILC capabilities for Higgs boson coupling measurements, arXiv:1207.2516 [INSPIRE].

[7] D.M. Asner et al., ILC Higgs white paper, arXiv:1310.0763 [INSPIRE].

[8] J. Ellis, P. Roloff, V. Sanz and T. You, Dimension-6 operator analysis of the CLIC sensitivity to new physics, JHEP 05 (2017) 096 [arXiv:1701.04804] [INSPIRE].

[9] Z. Chen et al., Cross section and Higgs mass measurement with Higgsstrahlung at the CEPC, Chin. Phys. C 41 (2017) 023003 [arXiv:1601.05352] [INSPIRE].

[10] Z. Kunszt and F. Zwirner, Testing the Higgs sector of the minimal supersymmetric standard model at large hadron colliders, Nucl. Phys. B 385 (1992) 3 [hep-ph/9203223] [INSPIRE].

[11] V.D. Barger, M.S. Berger, A.L. Stange and R.J.N. Phillips, Supersymmetric Higgs boson hadroproduction and decays including radiative corrections, Phys. Rev. D 45 (1992) 4128 [INSPIRE].

[12] H. Baer, M. Bisset, C. Kao and X. Tata, Observability of $\gamma \gamma$ decays of Higgs bosons from supersymmetry at hadron supercolliders, Phys. Rev. D 46 (1992) 1067 [INSPIRE].

[13] J.F. Gunion and L.H. Orr, Detecting the Higgs bosons of the minimal supersymmetric model, Phys. Rev. D 46 (1992) 2052 [inSPIRE].

[14] J.F. Gunion, H.E. Haber and C. Kao, Searching for the CP odd Higgs boson of the minimal supersymmetric model at hadron supercolliders, Phys. Rev. D 46 (1992) 2907 [INSPIRE].

[15] ATLAS collaboration, Supersymmetry searches, https://twiki.cern.ch/twiki/bin/view/AtlasPublic/SupersymmetryPublicResults.

[16] CMS collaboration, Supersymmetry searches, https://twiki.cern.ch/twiki/bin/view/CMSPublic/PhysicsResultsSUS.

[17] H.M. Lee, V. Sanz and M. Trott, Hitting sbottom in natural SUSY, JHEP 05 (2012) 139 [arXiv: 1204.0802] [INSPIRE].

[18] C. Grojean, E. Salvioni, M. Schlaffer and A. Weiler, Very boosted Higgs in gluon fusion, JHEP 05 (2014) 022 [arXiv:1312.3317] [INSPIRE].

[19] O. Brein and W. Hollik, Distributions for MSSM Higgs boson + jet production at hadron colliders, Phys. Rev. D 76 (2007) 035002 [arXiv:0705.2744] [InSPIRE].

[20] R.V. Harlander, S. Liebler and H. Mantler, SusHi: a program for the calculation of Higgs production in gluon fusion and bottom-quark annihilation in the Standard Model and the MSSM, Comput. Phys. Commun. 184 (2013) 1605 [arXiv: 1212.3249] [INSPIRE].

[21] R.V. Harlander, S. Liebler and H. Mantler, SusHi bento: beyond NNLO and the heavy-top limit, Comput. Phys. Commun. 212 (2017) 239 [arXiv:1605.03190] [INSPIRE]. 
[22] R. Harlander and P. Kant, Higgs production and decay: Analytic results at next-to-leading order QCD, JHEP 12 (2005) 015 [hep-ph/0509189] [INSPIRE].

[23] H. Mantler and M. Wiesemann, Hadronic Higgs production through NLO+PS in the SM, the 2HDM and the MSSM, Eur. Phys. J. C 75 (2015) 257 [arXiv:1504.06625] [INSPIRE].

[24] S. Dawson, I.M. Lewis and M. Zeng, Effective field theory for Higgs boson plus jet production, Phys. Rev. D 90 (2014) 093007 [arXiv: 1409.6299] [InSPIRE].

[25] D. Ghosh and M. Wiebusch, Dimension-six triple gluon operator in Higgs+jet observables, Phys. Rev. D 91 (2015) 031701 [arXiv:1411.2029] [InSPIRE].

[26] M. Grazzini, A. Ilnicka, M. Spira and M. Wiesemann, BSM effects on the Higgs transverse-momentum spectrum in an EFT approach, PoS(EPS-HEP 2015) 144 [arXiv: 1511.08059] [INSPIRE].

[27] S. Dawson, I.M. Lewis and M. Zeng, Usefulness of effective field theory for boosted Higgs production, Phys. Rev. D 91 (2015) 074012 [arXiv: 1501.04103] [InSPIRE].

[28] R. Edezhath, Dimension-6 operator constraints from boosted VBF Higgs, arXiv:1501.00992 [INSPIRE].

[29] M. Grazzini, A. Ilnicka, M. Spira and M. Wiesemann, Modeling BSM effects on the Higgs transverse-momentum spectrum in an EFT approach, JHEP 03 (2017) 115 [arXiv: 1612.00283] [INSPIRE].

[30] A. Banfi, A. Martin and V. Sanz, Probing top-partners in Higgs+jets, JHEP 08 (2014) 053 [arXiv:1308.4771] [INSPIRE].

[31] A. Azatov, M. Salvarezza, M. Son and M. Spannowsky, Boosting top partner searches in composite Higgs models, Phys. Rev. D 89 (2014) 075001 [arXiv:1308.6601] [INSPIRE].

[32] A. Azatov and A. Paul, Probing Higgs couplings with high $p_{T}$ Higgs production, JHEP 01 (2014) 014 [arXiv: 1309. 5273] [InSPIRE].

[33] J.F. Gunion and H.E. Haber, The CP conserving two Higgs doublet model: The Approach to the decoupling limit, Phys. Rev. D 67 (2003) 075019 [hep-ph/0207010] [INSPIRE].

[34] J.R. Ellis, M.K. Gaillard and D.V. Nanopoulos, A phenomenological profile of the Higgs boson, Nucl. Phys. B 106 (1976) 292 [INSPIRE].

[35] M.A. Shifman, A.I. Vainshtein, M.B. Voloshin and V.I. Zakharov, Low-energy theorems for Higgs boson couplings to photons, Sov. J. Nucl. Phys. 30 (1979) 711 [InSPIRE].

[36] A. Djouadi, Squark effects on Higgs boson production and decay at the LHC, Phys. Lett. B 435 (1998) 101 [hep-ph/9806315] [INSPIRE].

[37] ATLAS and CMS collaboration, Measurements of the Higgs boson production and decay rates and constraints on its couplings from a combined ATLAS and CMS analysis of the LHC pp collision data at $\sqrt{s}=7$ and 8 TeV, JHEP 08 (2016) 045 [arXiv: 1606.02266] [INSPIRE].

[38] B. Henning, X. Lu and H. Murayama, What do precision Higgs measurements buy us?, arXiv: 1404.1058 [INSPIRE].

[39] A. Drozd, J. Ellis, J. Quevillon and T. You, Comparing EFT and exact one-loop analyses of non-degenerate stops, JHEP 06 (2015) 028 [arXiv: 1504.02409] [INSPIRE].

[40] A. Drozd, J. Ellis, J. Quevillon and T. You, The universal one-loop effective action, JHEP 03 (2016) 180 [arXiv:1512.03003] [INSPIRE]. 
[41] J. Ellis, C.W. Murphy, V. Sanz and T. You, Updated global SMEFT fit to Higgs, diboson and electroweak data, JHEP 06 (2018) 146 [arXiv:1803.03252] [INSPIRE].

[42] S. Dawson, A. Djouadi and M. Spira, QCD corrections to SUSY Higgs production: the role of squark loops, Phys. Rev. Lett. 77 (1996) 16 [hep-ph/9603423] [INSPIRE].

[43] ATLAS and CMS collaboration, Combined measurement of the Higgs boson mass in pp collisions at $\sqrt{s}=7$ and 8 TeV with the ATLAS and CMS experiments, Phys. Rev. Lett. 114 (2015) 191803 [arXiv:1503.07589] [INSPIRE].

[44] J.R. Espinosa, C. Grojean, V. Sanz and M. Trott, NSUSY fits, JHEP 12 (2012) 077 [arXiv:1207.7355] [INSPIRE].

[45] U. Baur and E.W.N. Glover, Higgs boson production at large transverse momentum in hadronic collisions, Nucl. Phys. B 339 (1990) 38 [INSPIRE].

[46] G. Passarino and M.J.G. Veltman, One loop corrections for $e^{+} e^{-}$annihilation into $\mu^{+} \mu^{-}$in the Weinberg model, Nucl. Phys. B 160 (1979) 151 [InSPIRE].

[47] M. Muhlleitner, H. Rzehak and M. Spira, MSSM Higgs boson production via gluon fusion: the large gluino mass limit, JHEP 04 (2009) 023 [arXiv:0812.3815] [INSPIRE].

[48] G. Corcella et al., HERWIG 6: an event generator for hadron emission reactions with interfering gluons (including supersymmetric processes), JHEP 01 (2001) 010 [hep-ph/0011363] [INSPIRE].

[49] G.P. Salam and J. Rojo, A Higher Order Perturbative Parton Evolution Toolkit (HOPPET), Comput. Phys. Commun. 180 (2009) 120 [arXiv:0804.3755] [INSPIRE].

[50] A. Vogt, S. Moch and J.A.M. Vermaseren, The three-loop splitting functions in QCD: the singlet case, Nucl. Phys. B 691 (2004) 129 [hep-ph/0404111] [INSPIRE].

[51] S. Moch, J.A.M. Vermaseren and A. Vogt, The three loop splitting functions in QCD: the nonsinglet case, Nucl. Phys. B 688 (2004) 101 [hep-ph/0403192] [inSPIRE].

[52] T. Gehrmann and E. Remiddi, Numerical evaluation of two-dimensional harmonic polylogarithms, Comput. Phys. Commun. 144 (2002) 200 [hep-ph/0111255] [INSPIRE].

[53] M. Buza et al., Heavy quark coefficient functions at asymptotic values $Q^{2} \gg m^{2}$, Nucl. Phys. B 472 (1996) 611 [hep-ph/9601302] [InSPIRE].

[54] M. Buza, Y. Matiounine, J. Smith and W.L. van Neerven, Charm electroproduction viewed in the variable flavor number scheme versus fixed order perturbation theory, Eur. Phys. J. C 1 (1998) 301 [hep-ph/9612398] [INSPIRE].

[55] W.L. van Neerven and A. Vogt, NNLO evolution of deep inelastic structure functions: The Nonsinglet case, Nucl. Phys. B 568 (2000) 263 [hep-ph/9907472] [INSPIRE].

[56] W.L. van Neerven and A. Vogt, NNLO evolution of deep inelastic structure functions: the singlet case, Nucl. Phys. B 588 (2000) 345 [hep-ph/0006154] [INSPIRE].

[57] W.L. van Neerven and A. Vogt, Improved approximations for the three loop splitting functions in QCD, Phys. Lett. B 490 (2000) 111 [hep-ph/0007362] [INSPIRE].

[58] A.D. Martin, W.J. Stirling, R.S. Thorne and G. Watt, Parton distributions for the LHC, Eur. Phys. J. C 63 (2009) 189 [arXiv:0901.0002] [InSPIRE].

[59] ATLAS collaboration, Light-quark and gluon jet discrimination in pp collisions at $\sqrt{s}=7 \mathrm{TeV}$ with the ATLAS detector, Eur. Phys. J. C 74 (2014) 3023 [arXiv:1405.6583] [INSPIRE]. 
[60] L. Lönnblad, C. Peterson and T. Rognvaldsson, Finding gluon jets with a neural trigger, Phys. Rev. Lett. 65 (1990) 1321 [InSPIRE].

[61] J. Pumplin, How to tell quark jets from gluon jets, Phys. Rev. D 44 (1991) 2025 [InSPIRE].

[62] OPAL collaboration, P.D. Acton et al., A study of differences between quark and gluon jets using vertex tagging of quark jets, Z. Phys. C 58 (1993) 387 [INSPIRE].

[63] J. Gallicchio and M.D. Schwartz, Quark and gluon tagging at the LHC, Phys. Rev. Lett. 107 (2011) 172001 [arXiv:1106.3076] [INSPIRE].

[64] J. Gallicchio and M.D. Schwartz, Quark and gluon jet substructure, JHEP 04 (2013) 090 [arXiv: 1211.7038] [INSPIRE].

[65] D. Ferreira de Lima, P. Petrov, D. Soper and M. Spannowsky, Quark-gluon tagging with shower deconstruction: unearthing dark matter and Higgs couplings, Phys. Rev. D 95 (2017) 034001 [arXiv: 1607.06031] [inSPIRE].

[66] S.P. Jones, M. Kerner and G. Luisoni, Next-to-Leading-Order QCD corrections to Higgs boson plus jet production with full top-quark mass dependence, Phys. Rev. Lett. 120 (2018) 162001 [arXiv: 1802.00349] [INSPIRE].

[67] J.M. Lindert, K. Kudashkin, K. Melnikov and C. Wever, Higgs bosons with large transverse momentum at the LHC, Phys. Lett. B 782 (2018) 210 [arXiv:1801.08226] [InSPIRE].

[68] T. Neumann, NLO Higgs+jet at large transverse momenta including top quark mass effects, J. Phys. Comm. 2 (2018) 095017 [arXiv: 1802.02981] [InSPIRE].

[69] R. Boughezal et al., Higgs boson production in association with a jet at next-to-next-to-leading order, Phys. Rev. Lett. 115 (2015) 082003 [arXiv:1504.07922] [INSPIRE].

[70] X. Chen et al., NNLO QCD corrections to Higgs boson production at large transverse momentum, JHEP 10 (2016) 066 [arXiv:1607.08817] [INSPIRE].

[71] ATLAS collaboration, Measurements of the total and differential Higgs boson production cross sections combining the $H \rightarrow \gamma \gamma$ and $H \rightarrow Z Z * \rightarrow 4 \ell$ decay channels at $\sqrt{s}=8 \mathrm{TeV}$ with the ATLAS detector, Phys. Rev. Lett. 115 (2015) 091801 [arXiv:1504.05833] [INSPIRE].

[72] ATLAS and CMS collaborations, Measurements of the Higgs boson production and decay rates and constraints on its couplings from a combined ATLAS and CMS analysis of the LHC pp collision data at $\sqrt{s}=7$ and 8 TeV, ATLAS-CONF-2015-044 (2015).

[73] ATLAS collaboration, Combined measurements of Higgs boson production and decay in the $H \rightarrow Z Z^{*} \rightarrow 4 \ell$ and $H \rightarrow \gamma \gamma$ channels using $\sqrt{s}=13$ TeV pp collision data collected with the ATLAS experiment, ATLAS-CONF-2017-047 (2017).

[74] D. Barducci et al., Uncovering natural supersymmetry via the interplay between the LHC and direct dark matter detection, JHEP 07 (2015) 066 [arXiv:1504.02472] [INSPIRE].

[75] M. Schlaffer et al., Boosted Higgs shapes, Eur. Phys. J. C 74 (2014) 3120 [arXiv:1405.4295] [INSPIRE].

[76] R.V. Harlander and W.B. Kilgore, Next-to-next-to-leading order Higgs production at hadron colliders, Phys. Rev. Lett. 88 (2002) 201801 [hep-ph/0201206] [inSPIRE].

[77] H. Baer et al., The International Linear Collider technical design report - Volume 2: physics, arXiv: 1306.6352 [INSPIRE].

[78] M.L. Mangano and S.J. Parke, Multiparton amplitudes in gauge theories, Phys. Rept. 200 (1991) 301 [hep-th/0509223] [INSPIRE]. 
[79] L.J. Dixon, Calculating scattering amplitudes efficiently, hep-ph/9601359 [INSPIRE].

[80] B.A. Kniehl and M. Spira, Low-energy theorems in Higgs physics, Z. Phys. C 69 (1995) 77 [hep-ph/9505225] [INSPIRE].

[81] E. Bagnaschi, G. Degrassi, P. Slavich and A. Vicini, Higgs production via gluon fusion in the POWHEG approach in the SM and in the MSSM, JHEP 02 (2012) 088 [arXiv:1111.2854] [INSPIRE].

[82] S. Catani and M. Grazzini, An NNLO subtraction formalism in hadron collisions and its application to Higgs boson production at the LHC, Phys. Rev. Lett. 98 (2007) 222002 [hep-ph/0703012] [INSPIRE].

[83] M. Grazzini, NNLO predictions for the Higgs boson signal in the $H \rightarrow W W \rightarrow l \nu l \nu$ and $H \rightarrow Z Z \rightarrow 4 l$ decay channels, JHEP 02 (2008) 043 [arXiv: 0801.3232] [INSPIRE].

[84] C. Anastasiou, K. Melnikov and F. Petriello, Fully differential Higgs boson production and the di-photon signal through next-to-next-to-leading order, Nucl. Phys. B 724 (2005) 197 [hep-ph/0501130] [INSPIRE]. 